\title{
MIT
}

\section{HATS-47b, HATS-48Ab, HATS-49b, and HATS-72b: Four Warm Giant Planets Transiting K Dwarfs}

The MIT Faculty has made this article openly available. Please share how this access benefits you. Your story matters.

\begin{tabular}{|l|l|}
\hline As Published & $10.3847 / 1538-3881 /$ ab7821 \\
\hline Publisher & American Astronomical Society \\
\hline \hline Version & Final published version \\
\hline Citable link & https://hdl.handle.net/1721.1/136234 \\
\hline Terms of Use & $\begin{array}{l}\text { Article is made available in accordance with the publisher's } \\
\text { policy and may be subject to US copyright law. Please refer to the } \\
\text { publisher's site for terms of use. }\end{array}$ \\
\hline
\end{tabular}




\title{
HATS-47b, HATS-48Ab, HATS-49b, and HATS-72b: Four Warm Giant Planets Transiting K Dwarfs*
}

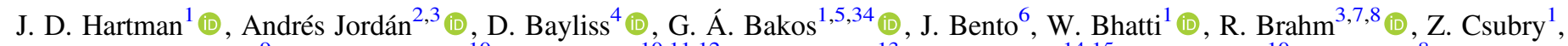

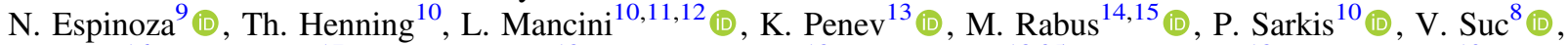

M. de Val-Borro ${ }^{16}$ (1D, G. Zhou ${ }^{17}$ (D), J. D. Crane ${ }^{18}$ (1) , S. Shectman ${ }^{18}$, J. K. Teske ${ }^{18,35}$, S. X. Wang ${ }^{18}$, R. P. Butler ${ }^{19}$ (1) , J. Lázár ${ }^{20}$, I. Papp $^{20}$, P. Sári ${ }^{20}$, D. R. Anderson ${ }^{4,21}$ (1) , C. Hellier ${ }^{21}$ (1), R. G. West ${ }^{4}$, K. Barkaoui ${ }^{22,23}$, F. J. Pozuelos ${ }^{22,24}$ (1), E. Jehin ${ }^{24}$, M. Gillon ${ }^{22}$ (1) L. Nielsen ${ }^{25}$, M. Lendl ${ }^{25,26}$ (1) , S. Udry ${ }^{25}$ (1) , George R. Ricker ${ }^{27}$, Roland Vanderspek ${ }^{27}$ (1), David W. Latham ${ }^{17}$ (1), S. Seager ${ }^{27,28,29}$, Joshua N. Winn ${ }^{1}$ (1) , Jessie Christiansen ${ }^{30}$ (1) Ian J. M. Crossfield ${ }^{27,31}$, Christopher E. Henze , $^{32}$, Jon M. Jenkins ${ }^{32}$ (1), Jeffrey C. Smith ${ }^{32,33}$ (1), and Eric B. Ting ${ }^{32}$

${ }^{1}$ Department of Astrophysical Sciences, Princeton University, NJ 08544, USA; jhartman @ astro.princeton.edu

${ }^{2}$ Facultad de Ingeniería y Ciencias, Universidad Adolfo Ibáñez, Av. Diagonal las Torres 2640, Peñalolén, Santiago, Chile ${ }^{3}$ Millennium Institute for Astrophysics, Chile

${ }^{4}$ Department of Physics, University of Warwick, Gibbet Hill Road, Coventry CV4 7AL, UK ${ }^{5}$ MTA Distinguished Guest Fellow, Konkoly Observatory, Hungary

${ }^{6}$ Research School of Astronomy and Astrophysics, Australian National University, Canberra, ACT 2611, Australia

${ }^{7}$ Center of Astro-Engineering UC, Pontificia Universidad Católica de Chile, Av. Vicuña Mackenna 4860, 7820436 Macul, Santiago, Chile

${ }^{8}$ Instituto de Astrofísica, Pontificia Universidad Católica de Chile, Av. Vicuña Mackenna 4860, 7820436 Macul, Santiago, Chile

${ }^{9}$ Space Telescope Science Institute, 3700 San Martin Drive, Baltimore, MD 21218, USA

${ }^{10}$ Max Planck Institute for Astronomy, Königstuhl 17, D-69117-Heidelberg, Germany

${ }^{11}$ Department of Physics, University of Rome Tor Vergata, Via della Ricerca Scientifica 1, I-00133-Roma, Italy

${ }^{12}$ INAF-Astrophysical Observatory of Turin, Via Osservatorio 20, I-10025-Pino Torinese, Italy

${ }^{13}$ Department of Physics, University of Texas at Dallas, Richardson, TX 75080, USA

${ }^{14}$ Las Cumbres Observatory Global Telescope Network, 6740 Cortona Dr. Suite 102, Goleta, CA 93117, USA

${ }^{15}$ Department of Physics, University of California, Santa Barbara, CA 93106-9530, USA

${ }^{16}$ Astrochemistry Laboratory, Goddard Space Flight Center, NASA, 8800 Greenbelt Rd, Greenbelt, MD 20771, USA

${ }^{17}$ Harvard-Smithsonian Center for Astrophysics, 60 Garden Street, Cambridge, MA 02138, USA

${ }^{18}$ The Observatories of the Carnegie Institution for Science, 813 Santa Barbara Street, Pasadena, CA 91101, USA

${ }^{19}$ Department of Terrestrial Magnetism, Carnegie Institution for Science, Washington, DC 20015, USA

${ }^{20}$ Hungarian Astronomical Association, 1451 Budapest, Hungary

${ }^{21}$ Astrophysics Group, Keele University, Staffordshire, ST5 5BG, UK

${ }^{22}$ Astrobiology Research Unit, University of Liege, Allée du 6 Août 19C, B-4000 Liège, Belgium

${ }^{23}$ LPHEA Laboratory, Oukaimeden Observatory, Cadi Ayyad University/FSSM, BP 2390, Marrakesh, Morocco

${ }^{24}$ STAR Research Unit, University of Liege, Allée du 6 Août 19C, B-4000 Liège, Belgium

${ }^{25}$ Observatoire de Genève, Université de Genève, $51 \mathrm{Ch}$. des Maillettes, 1290 Sauverny, Switzerland

${ }^{26}$ Space Research Institute, Austrian Academy of Sciences, Schmiedlstr. 6, A-8042 Graz, Austria

${ }^{27}$ Department of Physics and Kavli Institute for Astrophysics and Space Research, Massachusetts Institute of Technology, Cambridge, MA 02139, USA

${ }^{28}$ Department of Earth, Atmospheric and Planetary Sciences, Massachusetts Institute of Technology, Cambridge, MA 02139, USA

${ }^{29}$ Department of Aeronautics and Astronautics, MIT, 77 Massachusetts Avenue, Cambridge, MA 02139, USA

${ }^{30}$ Caltech/IPAC-NASA Exoplanet Science Institute, 770 S. Wilson Avenue, Pasadena, CA 91106, USA

${ }^{31}$ Department of Physics and Astronomy, University of Kansas, 1251 Wescoe Hall Dr., Lawrence, KS 66045, USA

${ }^{32}$ NASA Ames Research Center, Moffett Field, CA, 94035, USA

${ }^{33}$ SETI Institute, Mountain View, CA 94043, USA

Received 2020 January 9; revised 2020 February 10; accepted 2020 February 13; published 2020 March 26

\begin{abstract}
We report the discovery of four transiting giant planets around K dwarfs. The planets HATS-47b, HATS-48Ab, HATS$49 \mathrm{~b}$, and HATS-72b have masses of $0.369_{-0.021}^{+0.031} M_{\mathrm{J}}, 0.243_{-0.030}^{+0.022} M_{\mathrm{J}}, 0.353_{-0.027}^{+0.038} M_{\mathrm{J}}$, and $0.1254 \pm 0.0039 M_{\mathrm{J}}$, respectively, and radii of $1.117 \pm 0.014 R_{\mathrm{J}}, 0.800 \pm 0.015 R_{\mathrm{J}}, \quad 0.765 \pm 0.013 R_{\mathrm{J}}$, and $0.7224 \pm 0.0032 R_{\mathrm{J}}$, respectively. The planets orbit close to their host stars with orbital periods of 3.9228 days, 3.1317 days, 4.1480 days, and 7.3279 days, respectively. The hosts are main-sequence $\mathrm{K}$ dwarfs with masses of $0.674_{-0.012}^{+0.016} M_{\odot}, 0.7279 \pm$ $0.0066 M_{\odot}, 0.7133 \pm 0.0075 M_{\odot}$, and $0.7311 \pm 0.0028$, and with $V$-band magnitudes of $V=14.829 \pm 0.010$, $14.35 \pm 0.11,14.998 \pm 0.040$ and $12.469 \pm 0.010$. The super-Neptune HATS-72b (a.k.a. WASP-191b and TOI
\end{abstract}

\footnotetext{
* The HATSouth network is operated by a collaboration consisting of Princeton University (PU), the Max Planck Institute für Astronomie (MPIA), the Australian National University (ANU), and the Pontificia Universidad Católica de Chile (PUC). The station at Las Campanas Observatory (LCO) of the Carnegie Institute is operated by PU in conjunction with PUC, the station at the High Energy Spectroscopic Survey (H.E.S.S.) site is operated in conjunction with MPIA, and the station at Siding Spring Observatory (SSO) is operated jointly with ANU. Based in part on observations made with the MPG $2.2 \mathrm{~m}$ Telescope at the ESO Observatory in La Silla. Based on observations collected at the European Southern Observatory. This paper includes data gathered with the 6.5 meter Magellan Telescopes at Las Campanas Observatory, Chile.

${ }^{34}$ Packard Fellow.

35 NASA Hubble Fellow.
} 
294.01) was independently identified as a transiting planet candidate by the HATSouth, WASP, and TESS surveys, and we present a combined analysis of all of the data gathered by each of these projects (and their follow-up programs). An exceptionally precise mass is measured for HATS-72b thanks to high-precision radial velocity (RV) measurements obtained with VLT/ESPRESSO, FEROS, HARPS, and Magellan/PFS. We also incorporate TESS observations of the warm Saturn-hosting systems HATS-47 (a.k.a. TOI 1073.01), HATS-48A, and HATS-49. HATS-47 was independently identified as a candidate by the TESS team, while the other two systems were not previously identified from the TESS data. The RV orbital variations are measured for these systems using Magellan/PFS. HATS-48A has a resolved 5!" 4 neighbor in Gaia DR2, which is a common-proper-motion binary star companion to HATS-48A with a mass of $0.22 M_{\odot}$ and a current projected physical separation of $\sim 1400$ au.

Unified Astronomy Thesaurus concepts: Exoplanets (498); Extrasolar gas giants (509); Hot Jupiters (753); Transits (1711)

Supporting material: machine-readable tables

\section{Introduction}

Much of our empirical knowledge about the physical properties of planets beyond the solar system (exoplanets) comes from observing planets with orbits that are fortuitously oriented such that the planets transit in front of their host stars from our vantage point.

Of particular importance are transiting planets with masses that have been measured either via high-precision radial velocity (RV) observations (e.g., Henry et al. 2000; Charbonneau et al. 2000; Konacki et al. 2003), or by observing deviations from strict periodicity in the transit times of other planets in the planetary system (e.g., Holman et al. 2010). Measuring both the planetary mass and radius (the latter being measurable from the transits once the physical properties of the host star are determined), together with the incident stellar flux (determined from the period once the stellar luminosity and mass are known) and system age (determined as one of the host star properties), allows constraints to be set on the composition of the planet (e.g., Guillot et al. 2006). The necessary stellar properties can be determined by comparing photometric, astrometric, and spectroscopic observations of the star to empirical or theoretical relations between stellar physical and observable parameters.

To date, more than 3,000 transiting planets have been confirmed or validated. ${ }^{36}$ About $90 \%$ of these were found by the NASA Kepler mission (Borucki et al. 2010), or its successor $K 2$ (Howell et al. 2014). However, the planetary masses have not yet been measured for the majority of these. Of the $\sim 600$ transiting planets with measured masses, only about one-third were discovered by Kepler or K2. The masses of typical Kepler and $K 2$ transiting planets are difficult to measure because the planets are too small, have periods that are too long, or are orbiting stars that are too faint for effective RV monitoring.

In order to increase the number of exoplanets-particularly planets smaller than Neptune-with measured masses, NASA launched the TESS space-based photometer (Ricker et al. 2015). For its primary mission, TESS is carrying out a twoyear survey of approximately three-quarters of the sky to find transiting planets around bright stars. As of the time of writing, the mission has been operational for a year and a half, and has led to the identification of about 1000 transiting planet candidates, of which 17 new planets have so far been confirmed and have had their masses measured. The follow-up observations needed to confirm and characterize the transiting planet candidates identified by TESS are being

\footnotetext{
36 The NASA Exoplanet Archive, https://exoplanetarchive.ipac.caltech.edu, accessed 2019 September 9.
}

carried out by the community, with organization provided by the TESS Follow-up Program (TFOP; Collins et al. 2018).

Approximately half of the transiting planets with measured masses were discovered by wide-field ground-based transit surveys, especially the WASP (Pollacco et al. 2006), HAT (Bakos et al. 2004, 2013), and KELT (Pepper et al. 2007, 2012) projects. These projects have primarily been sensitive to shortperiod gas-giant planets. They have discovered the majority of exoplanets that have been the subject of observational studies of the planetary atmospheres, as well as measurements of the stellar-spin-planetary-orbit alignment. These projects, some of which have been in operation for more than a decade, are now contributing to the follow-up and confirmation of transiting planets from the TESS mission. This includes both providing photometric observations carried out with the ground-based transit survey instruments, and follow-up spectroscopic and photometric observations using facilities, procedures, and teams that were originally brought together to confirm candidates from the ground-based surveys.

Many of the transiting giant planet candidates detected by TESS had already been identified by the ground-based projects. Some are confirmed and published planets, some have already been ruled out as false positives, some have been confirmed but not yet published (including two cases presented in this paper), some have survived initial followup vetting observations but are not yet confirmed, and some have simply not been followed up yet. There are also cases in which objects that were selected as candidates by the groundbased surveys have not been identified publicly by the TESS team. Many of these latter objects are false alarms. However, in some cases, the transiting planets have been confirmed, and the reason they were not identified by TESS is that the signals are weak due to crowding, substantial scattered light, or other issues. In other cases, the candidates are around stars that are fainter than the magnitude thresholds being applied by the TESS team in searching for transit signals. Two of the planets presented in this paper have stars that are fainter than the magnitude limits currently being searched with the standard TESS procedures.

In this paper, we present the discovery and characterization —including precise mass measurements — of four giant planets transiting K dwarf stars. The four planets, HATS-47b, HATS$48 \mathrm{Ab}$, HATS-49b, and HATS-72b, were identified by the HATSouth transit survey, and one was also independently identified by the WASP survey. All four of these objects also have transits that can be detected using TESS data, but only two of them have been selected as candidate transiting planet systems by the TESS team. The planets have masses between 
$0.1254 \pm 0.0039 M_{\mathrm{J}}$ (HATS-72b) and $0.369_{-0.021}^{+0.031} M_{\mathrm{J}}$ (HATS$47 \mathrm{~b}$ ), radii between $0.7224 \pm 0.0032 R_{\mathrm{J}}$ (HATS-72b) and $1.117 \pm 0.014 R_{\mathrm{J}}$ (HATS-47b), and orbit stars with masses between $0.674_{-0.012}^{+0.016} M_{\odot}$ (HATS-47) and $0.7311 \pm 0.0028 M_{\odot}$ (HATS-72). Thanks to the relatively low masses and luminosities of these host stars, the planets can all be classified as "warm" giant planets rather than "hot" giant planets: in all four cases, the expected equilibrium temperature-computed under the assumptions of zero albedo and isotropic reradiation -is below $1000 \mathrm{~K}$. While hot giant planets exhibit a radius inflation anomaly (e.g., Hartman et al. 2011, among many other examples), warm giant planets are observed to have radii that are consistent with theoretical expectations (e.g., Kovács et al. 2010). More recently, Sestovic et al. (2018) have argued that planets with masses $M_{p}<0.37 M_{\mathrm{J}}$, which includes all the planets here, do not exhibit anomalous radius inflation, even when highly irradiated. As a result, it is reasonable to suppose that meaningful inferences about the bulk planet metal content can be derived for these warm giant planets (e.g., Thorngren et al. 2016). This makes warm low-mass giant planets, like those presented here, particularly useful for testing theories of giant planet formation and structure.

In the following section, we discuss the observations used to detect, confirm, and characterize the transiting planet systems HATS-47, HATS-48A, HATS-49, and HATS-72. The analysis of these data is described in Section 3. We discuss the results in Section 4.

\section{Observations}

Figures 1-4 show some of the observations collected for HATS-47, HATS-48A, HATS-49, and HATS-72, respectively. Each figure shows the HATSouth light curve used to detect the transits, the ground-based follow-up transit light curves, the high-precision RVs and spectral line bisector spans (BSs), and the catalog broad-band photometry, including parallax corrections from Gaia DR2 (Gaia Collaboration et al. 2018), used in characterizing the host stars. We also show the TESS light curves for each system in Figures 5-8, and the WASP light curve for HATS-72 in Figure 9. Below, we describe the observations of these objects that were collected and analyzed here.

\subsection{Photometric Detection}

All four of the systems presented here were detected as transiting planet candidates by the HATSouth ground-based transiting planet survey (Bakos et al. 2013) as we discuss in Section 2.1.1. The transits of all four objects are also detected in time-series observations collected by the TESS mission (Section 2.1.2), and HATS-47b and HATS-72b were independently identified as transiting planet candidates by the TESS team based on these data. The transits of HATS-72b were also independently identified by the WASP project, as discussed in Section 2.1.3.

\subsubsection{HATSouth}

HATSouth uses a network of 24 telescopes, each $0.18 \mathrm{~m}$ in aperture, and $4 \mathrm{~K} \times 4 \mathrm{~K}$ front-side-illuminated CCD cameras. These are attached to a total of six fully automated mounts, each with an associated enclosure, which are in turn located at three sites around the Southern hemisphere. The three sites are
Las Campanas Observatory in Chile, the site of the H.E.S.S. gamma-ray observatory in Namibia, and Siding Spring Observatory in Australia. The operations and observing procedures of the network were described by Bakos et al. (2013), while the methods for reducing the images to trendfiltered light curves and searching for candidate transiting planets were described by Penev et al. (2013). We note that the trend filtering makes use of the Trend-filtering Algorithm of Kovács et al. (2005), while transit signals are detected using the Box-fitting Least Squares (BLS) method of Kovács et al. (2002). The HATSouth observations of each system are summarized in Table 1, and displayed in Figures 1-4, while the light-curve data are made available in Table 2.

We also searched the HATSouth light curves for other periodic signals using the Generalized Lomb-Scargle method (GLS; Zechmeister \& Kürster 2009), and for additional transit signals by applying a second iteration of BLS. Both of these searches were performed on the residual light curves after subtracting the best-fit transit models.

HATS-47 shows quasi-sinusoidal periodic variability at a period of $6.621581 \pm 0.000078$ days and semi-amplitude of $1.96 \pm 0.18 \mathrm{mmag}$ in the $r^{\prime}$ band (Figure 10). The GLS false alarm probability, calibrated using a bootstrap procedure, is $10^{-34}$, indicating a strong detection. We interpret this signal as the photometric rotation period of the star. BLS also picks up on this modulation as the strongest "transit-like" signal in the residuals, though the duration of the "transit" feature is much too long for this to be due to the transit of a planet or star. No other notable transit signals are seen in the light curve.

No periodic signals are detected in the light curves of HATS48A or HATS-49.

For HATS-72, GLS identifies a periodic signal at a period of $P=48.725 \pm 0.015$ days and with a semi-amplitude of $0.52 \pm 0.12 \mathrm{mmag}$ (Figure 10). We estimate a bootstrapcalibrated false alarm probability of $10^{-4}$, indicating that this is a marginal detection, though we list this as our best estimate for the photometric rotation period of the star. We do not find evidence with BLS for any additional transit signals in our light curve of this object.

\subsubsection{TESS}

All four systems were observed by the NASA TESS mission as summarized in Table 1.

HATS-47 was observed in short-cadence mode through an approved TESS Guest-Investigator program (G011214; PI Bakos) to observe HATSouth transiting planet candidates with TESS. The short-cadence observations were reduced to light curves by the NASA Science Processing Operations Center (SPOC) Pipeline at NASA Ames Research Center (Jenkins et al. 2010; Jenkins et al. 2016). Two threshold crossing events were identified by this pipeline for this target, and the object was selected as a transiting planet candidate and assigned the TESS Object of Interest (TOI) identifier TOI 1073.01 by the TESS Science Office team after inspecting the data validation report produced by the SPOC pipeline. The target passed all of the data validation tests conducted by the pipeline, including no discernible difference between odd and even transits, no evidence for a weak secondary event, no evidence for stronger transits in a halo aperture compared to the optimal aperture used to extract the light curve, strong evidence that the target is not a false alarm due to correlated noise, and no evidence for 

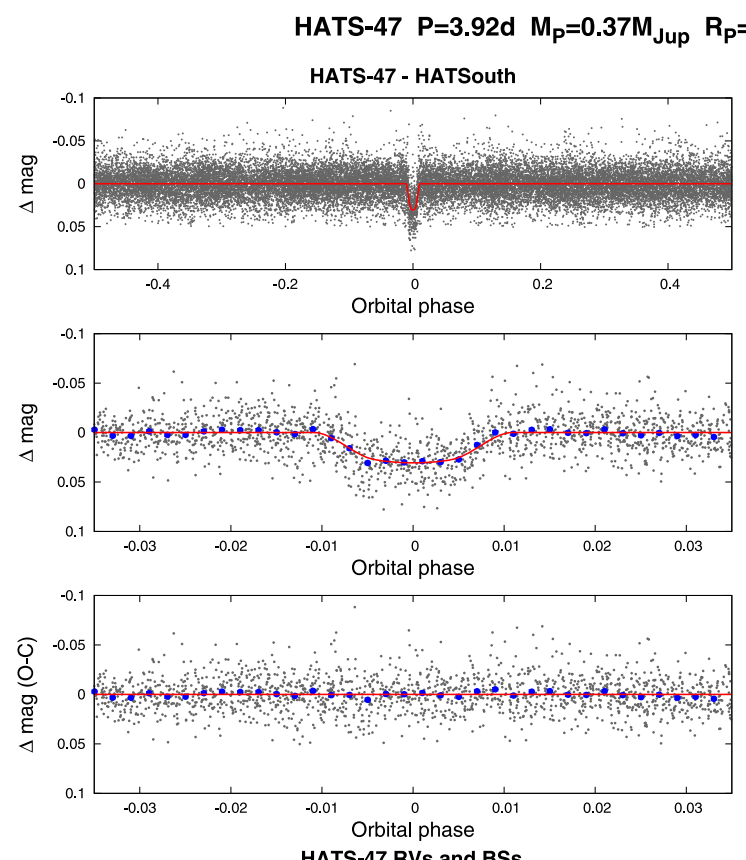

$=1.11 R_{\text {Jup }} M_{S}=0.69 M_{\text {Sun }} R_{S}=0.66 R_{\text {Sun }}$
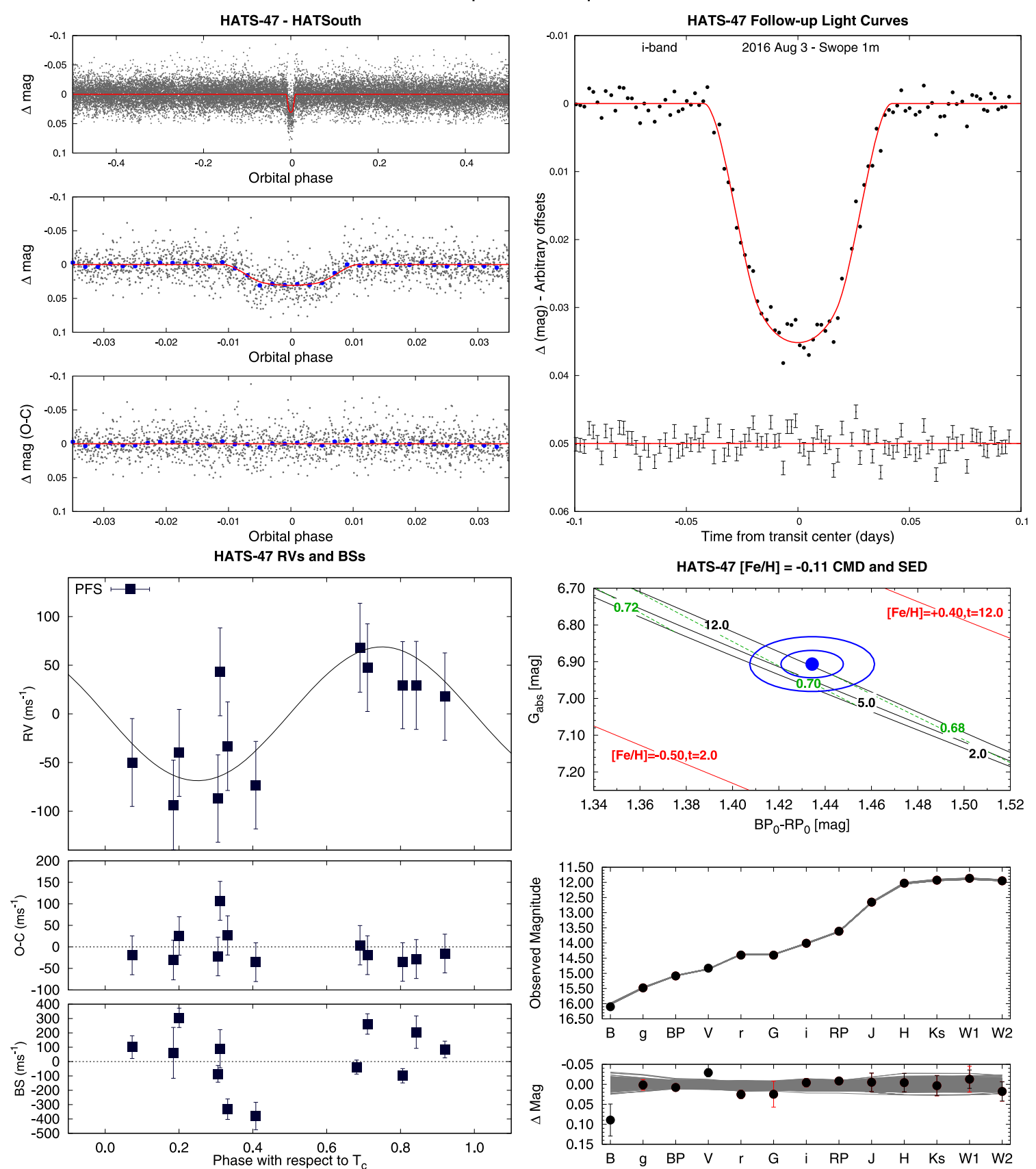

Figure 1. Observations used to confirm the transiting planet system HATS-47, excluding data from the NASA TESS mission, which are shown in Figure 5. Top left: phase-folded unbinned HATSouth light curve. Top panel shows the full light curve, middle panel shows the light curve zoomed-in on the transit, and bottom panel shows the residuals from the best-fit model zoomed-in on the transit. Solid lines show the model fits to the light curves. Dark filled circles show the light curves binned in phase with a bin size of 0.002. Top right: unbinned follow-up transit light curves corrected for instrumental trends fitted simultaneously with the transit model, which is overplotted. Dates, filters, and instruments used are indicated. In this figure, the residuals are shown below the light curve. In Figures $2-4$, the residuals are shown on the right-hand side in the same order as the original light curves. Error bars represent the photon and background shot noise, plus the readout noise. Note that these uncertainties are scaled up in the fitting procedure to achieve a reduced $\chi^{2}$ of unity, but the uncertainties shown in the plot have not been scaled. Bottom left: high-precision RVs phased with respect to the mid-transit time. The instruments used are labeled in the plot. Top panel shows the phased measurements together with the best-fit model. Center-of-mass velocity has been subtracted. Second panel shows the velocity $O-C$ residuals. Error bars include the estimated jitter. Third panel shows the bisector spans. Bottom right: color-magnitude diagram (CMD) and spectral energy distribution (SED). Top panel shows the absolute $G$ magnitude vs. the dereddened $B P-R P$ color compared to theoretical isochrones (black lines) and stellar evolution tracks (green lines) from the PARSEC models interpolated at the best-estimate value for the metallicity of the host. Age of each isochrone is listed in black in Gyr, while mass of each evolution track is listed in green in solar masses. Solid red lines show isochrones at higher and lower metallicities than the best-estimate value, with the metallicity and age in Gyr of each isochrone labeled on the plot. Filled blue circles show the measured reddening- and distance-corrected values from Gaia DR2, while blue lines indicate the $1 \sigma$ and $2 \sigma$ confidence regions, including the estimated systematic errors in the photometry. Middle panel shows the SED as measured via broadband photometry through the listed filters. Here, we plot the observed magnitudes without correcting for distance or extinction. Overplotted are 200 model SEDs randomly selected from the MCMC posterior distribution produced through the global analysis (gray lines). Model makes use of the predicted absolute magnitudes in each bandpass from the PARSEC isochrones, distance to the system (constrained largely via Gaia DR2), and extinction (constrained from the SED with a prior coming from the MWDUST 3D Galactic extinction model). Bottom panel shows the $O-C$ residuals from the best-fit model SED. 

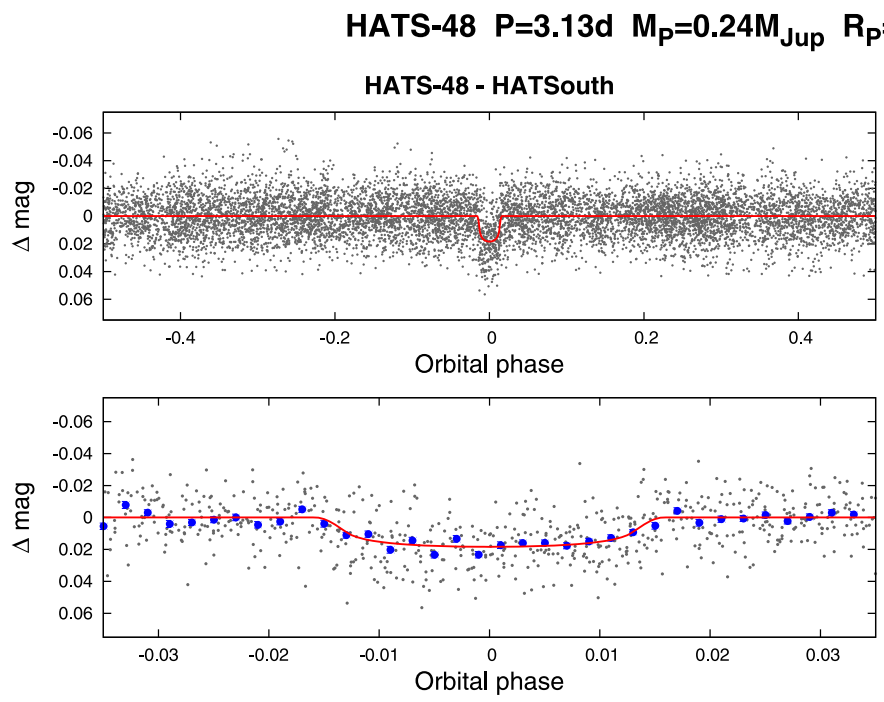

$R_{P}=0.80 R_{\text {Jup }} M_{S}=0.73 M_{\text {Sun }} R_{S}=0.72 R_{\text {Sun }}$
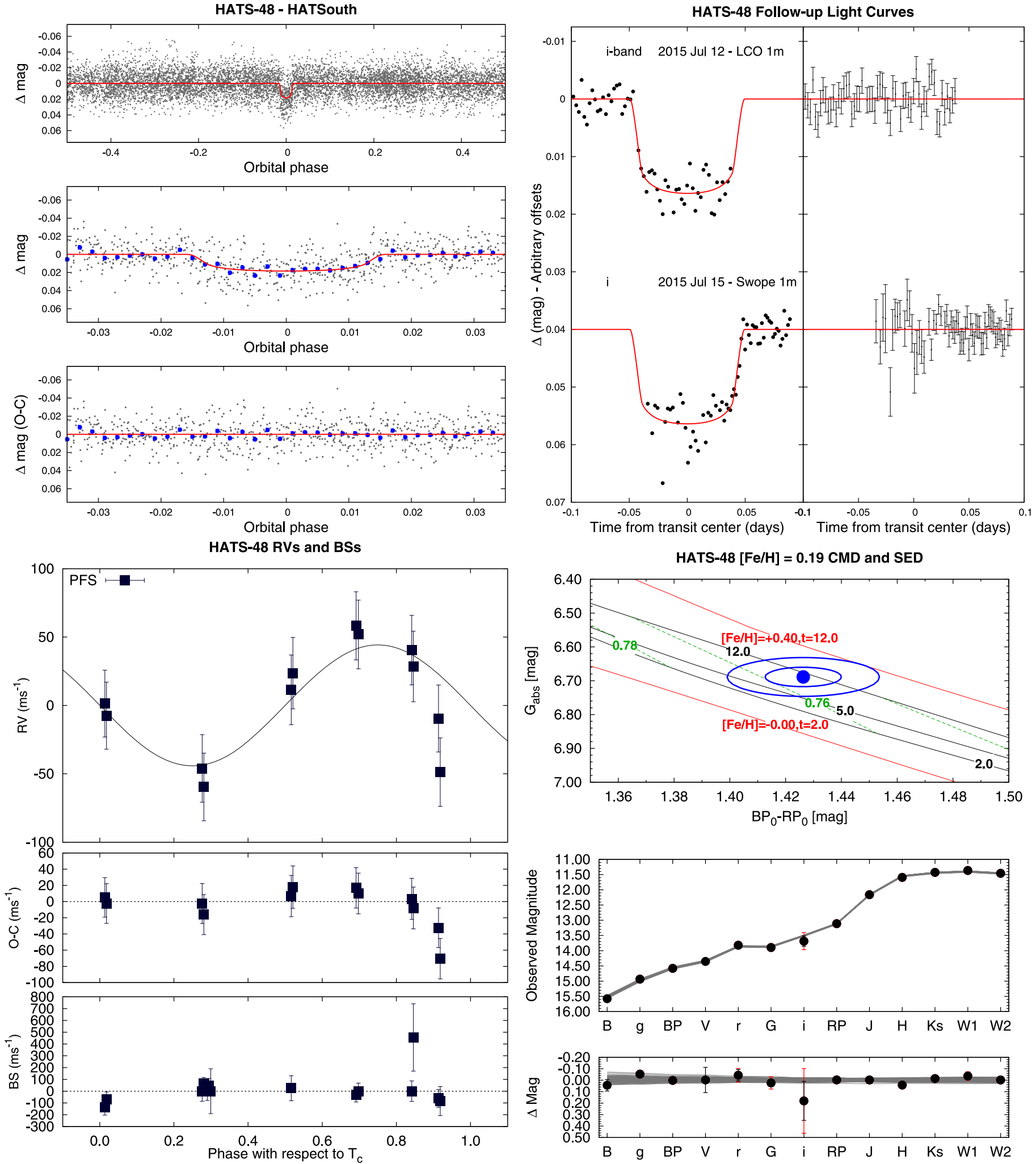

Figure 2. Same as Figure 1, but here we show the observations of HATS-48A together with our best-fit model. TESS light curve for this system is shown in Figure 6.

variations in the difference image centroid. We obtained the SPOC PDC light curve (Smith et al. 2012; Stumpe et al. 2014) for HATS-47 from the Barbara A. Mikulski Archive for Space Telescopes.
HATS-47 is blended in the TESS images with two other comparably bright stars (the two neighbors are separated from HATS- 47 by $33^{\prime \prime}$ and $42^{\prime \prime}$ ). These neighbors are both wellresolved by HATSouth and the facility used for photometric 

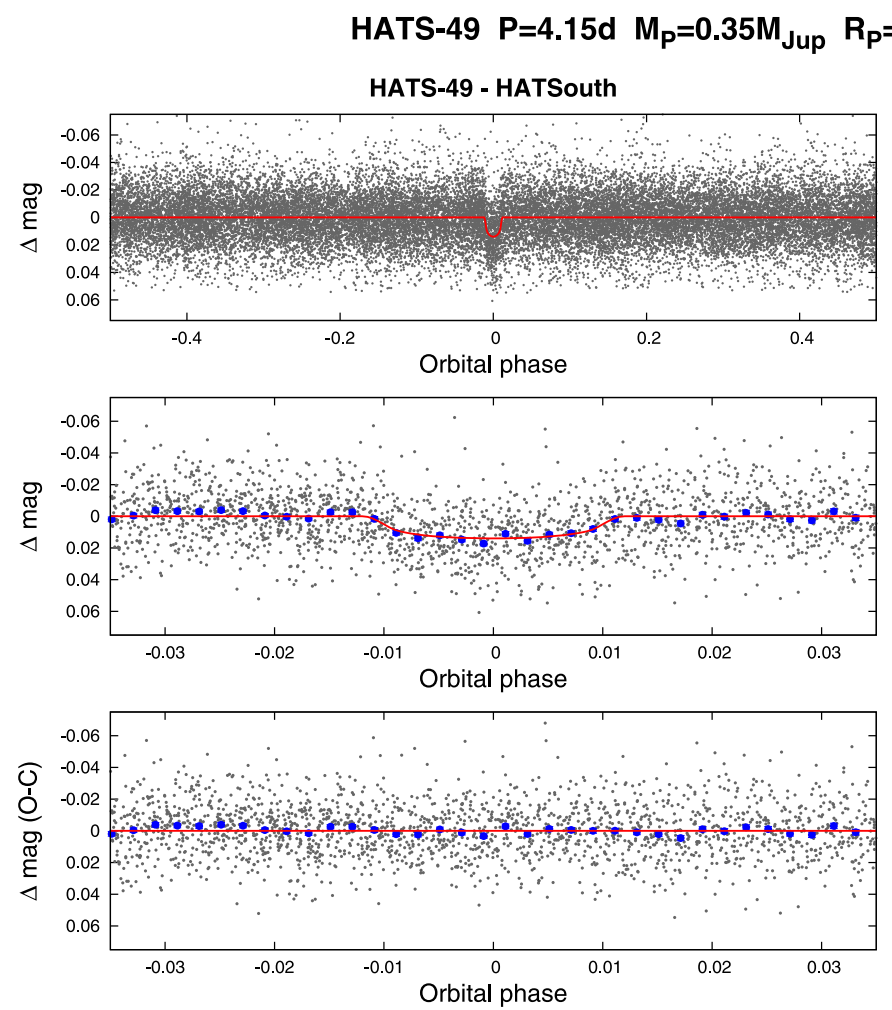

HATS-49 RVs and BSs
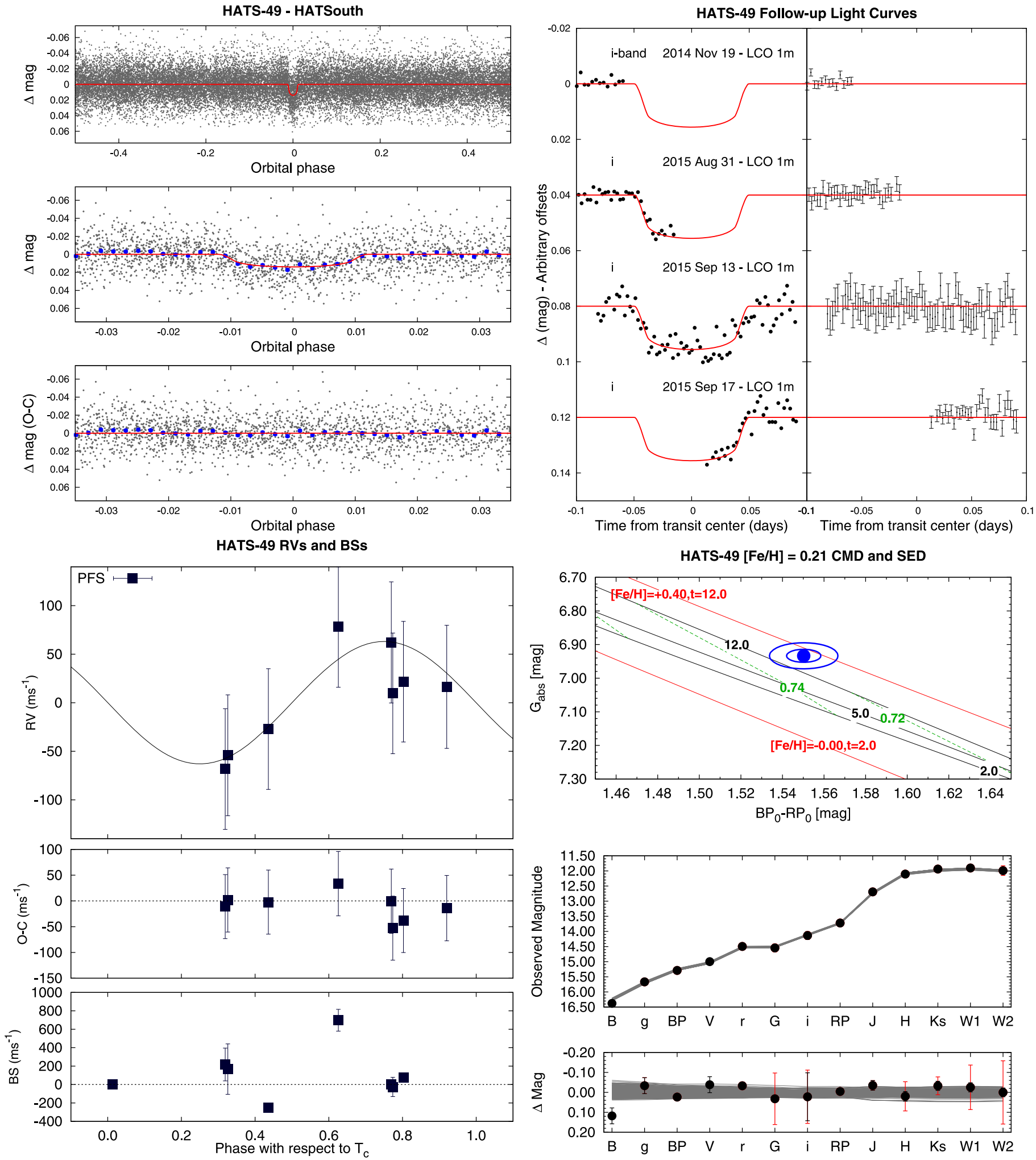

Figure 3. Same as Figure 1, but here we show the observations of HATS-49 together with our best-fit model. TESS light curve for this system is shown in Figure 7.

follow-up observations (Section 2.3). A correction for dilution is applied in the PDC process, but may have been overestimated in this case, leading to a slightly deeper transit seen in the TESS light curve (Figure 5) compared to the other light curves (Figure 1).
The TESS light curve of HATS-47 shows a clear quasisinusoidal out-of-transit variability with a period of $6.22158 \pm$ 0.00011 days and a semi-amplitude of $5.79 \pm 0.33 \mathrm{mmag}$. The period is close to, but different from, the rotation period of 

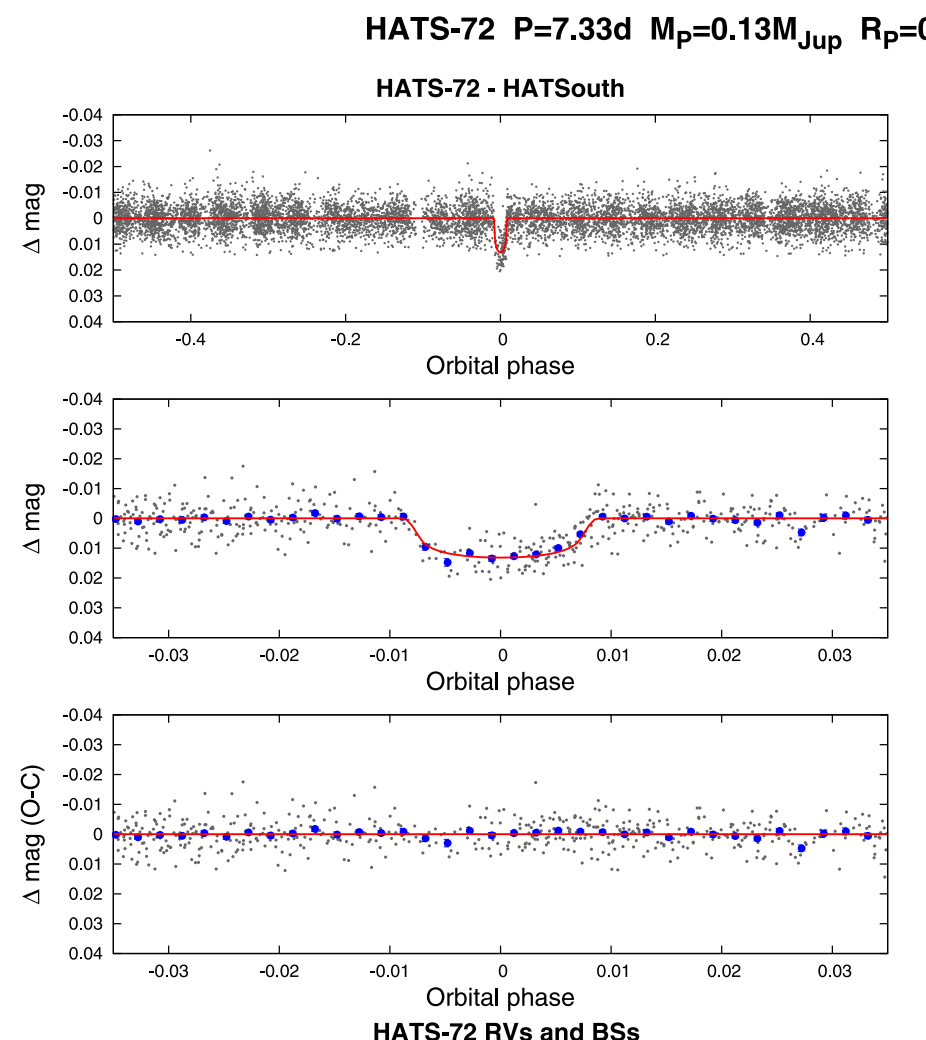

$=0.72 R_{\text {Jup }} M_{S}=0.73 M_{\text {Sun }} R_{S}=0.72 R_{\text {Sun }}$
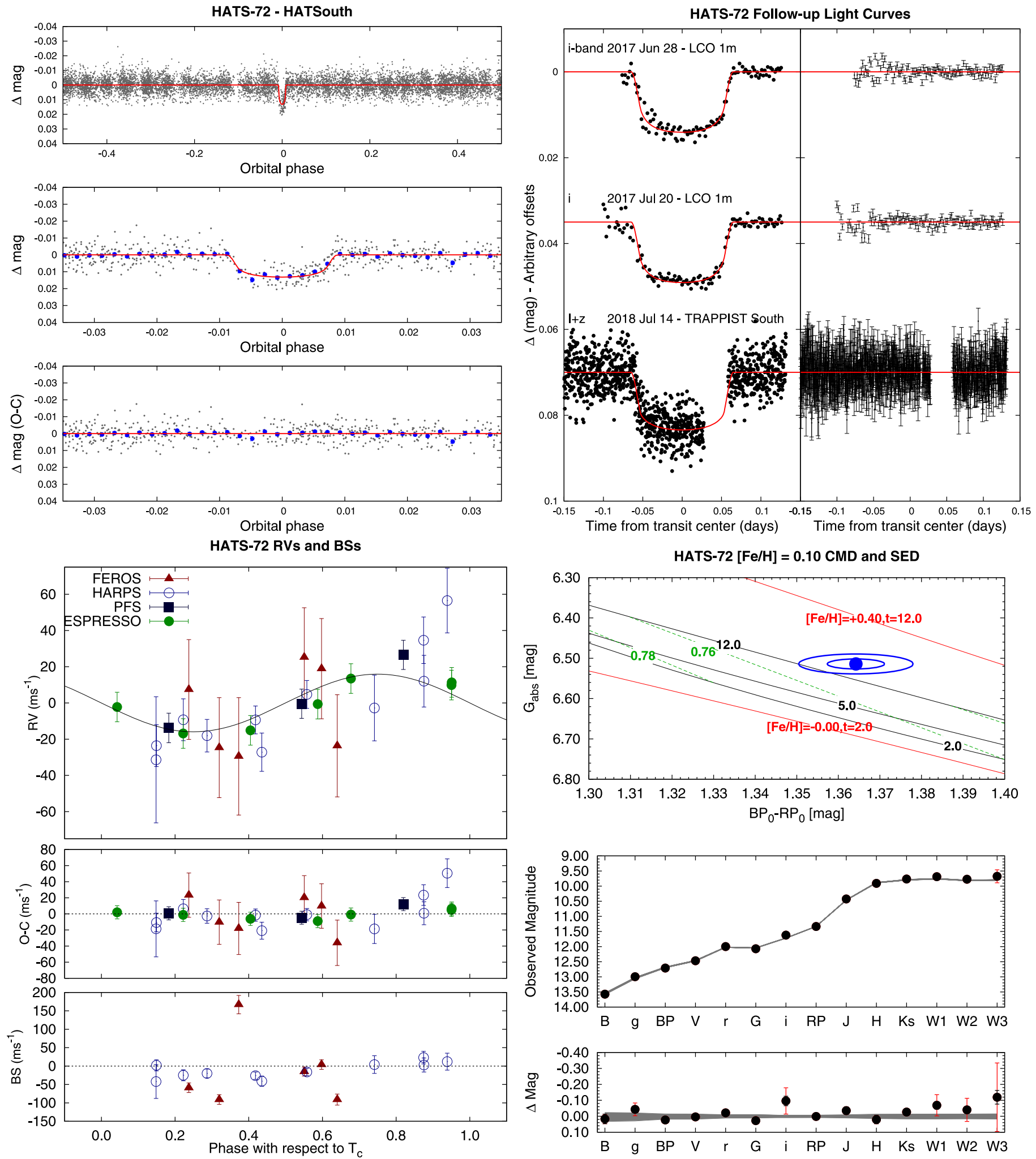

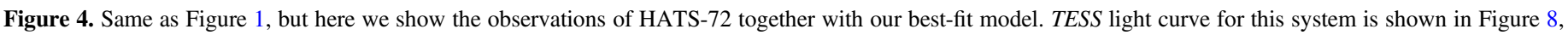
while the WASP light curve is shown in Figure 9.

$6.621581 \pm 0.000078$ days estimated independently from the HATSouth light curve. The difference is presumably a result of starspot evolution and/or differential rotation. We take the average of these two measurements as our estimate for the photometric rotation period of the star ( $6.42 \pm 0.28$ days). We filtered the quasi-sinusoidal variation out of the TESS light curve 

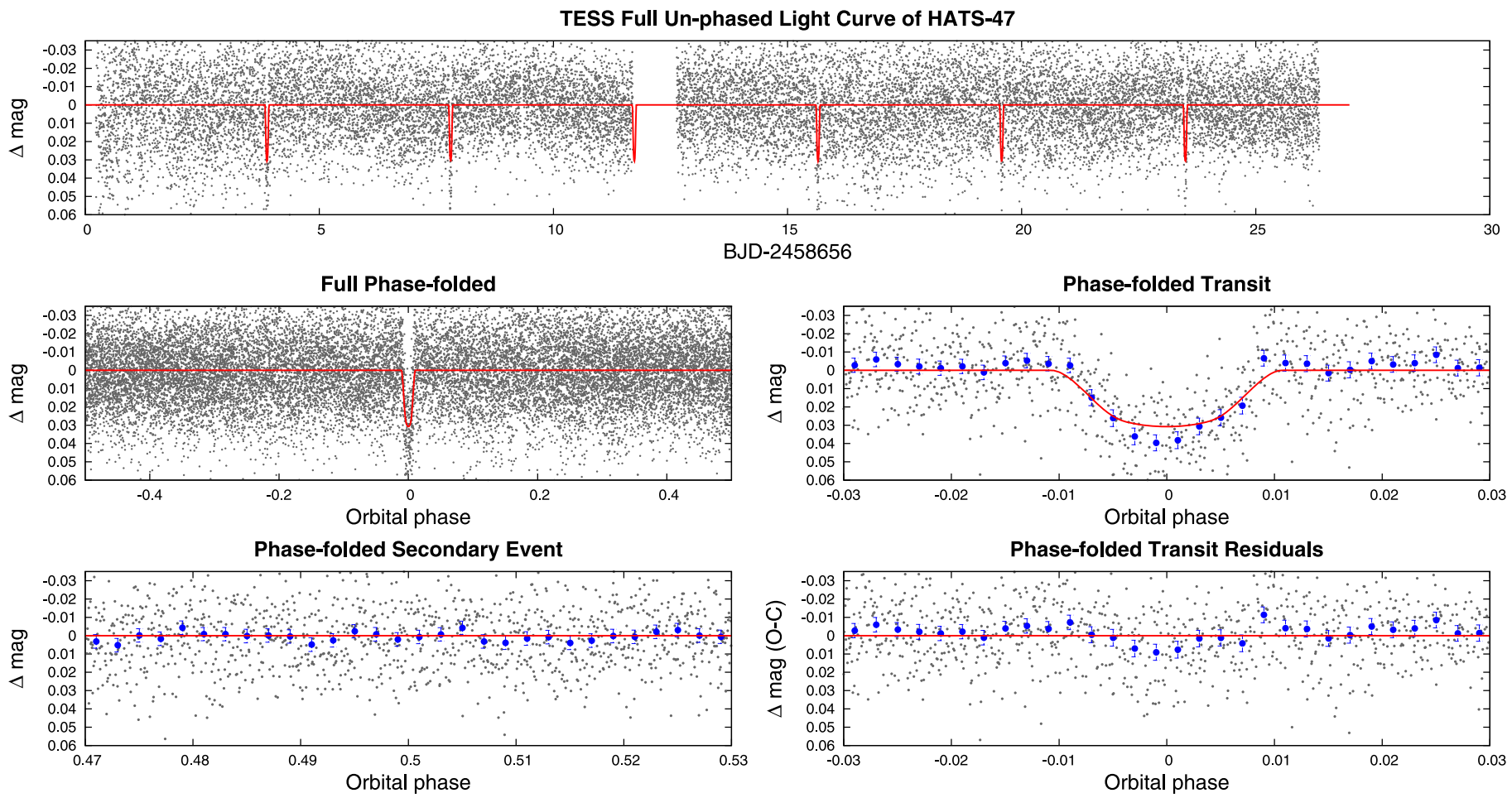

Figure 5. TESS short-cadence light curve for HATS-47. We show the full unphased light curve as a function of time (top), the full phase-folded light curve (middle left), the phase-folded light curve zoomed-in on the planetary transit (middle right), the phase-folded light curve zoomed-in on the secondary eclipse (bottom left), and the residuals from the best-fit model, phase-folded and zoomed-in on the planetary transit (bottom right). Solid line in each panel shows the model fit to the light curve. Dark filled circles show the light curve binned in phase with a bin size of 0.002. Other observations included in our analysis of this system are shown in Figure 1. TESS light curve has been corrected for dilution from neighbors as part of the PDC process, but in this case, where two bright neighbors are blended with the target in the TESS images, the correction appears to have been somewhat overestimated. This leads to a slightly deeper apparent transit in the TESS PDC light curve compared to the model. The model, however, fits the HATSouth and Swope $1 \mathrm{~m}$ transit observations (Figure 1), which were obtained at sufficiently high spatial resolution to resolve the neighbors.
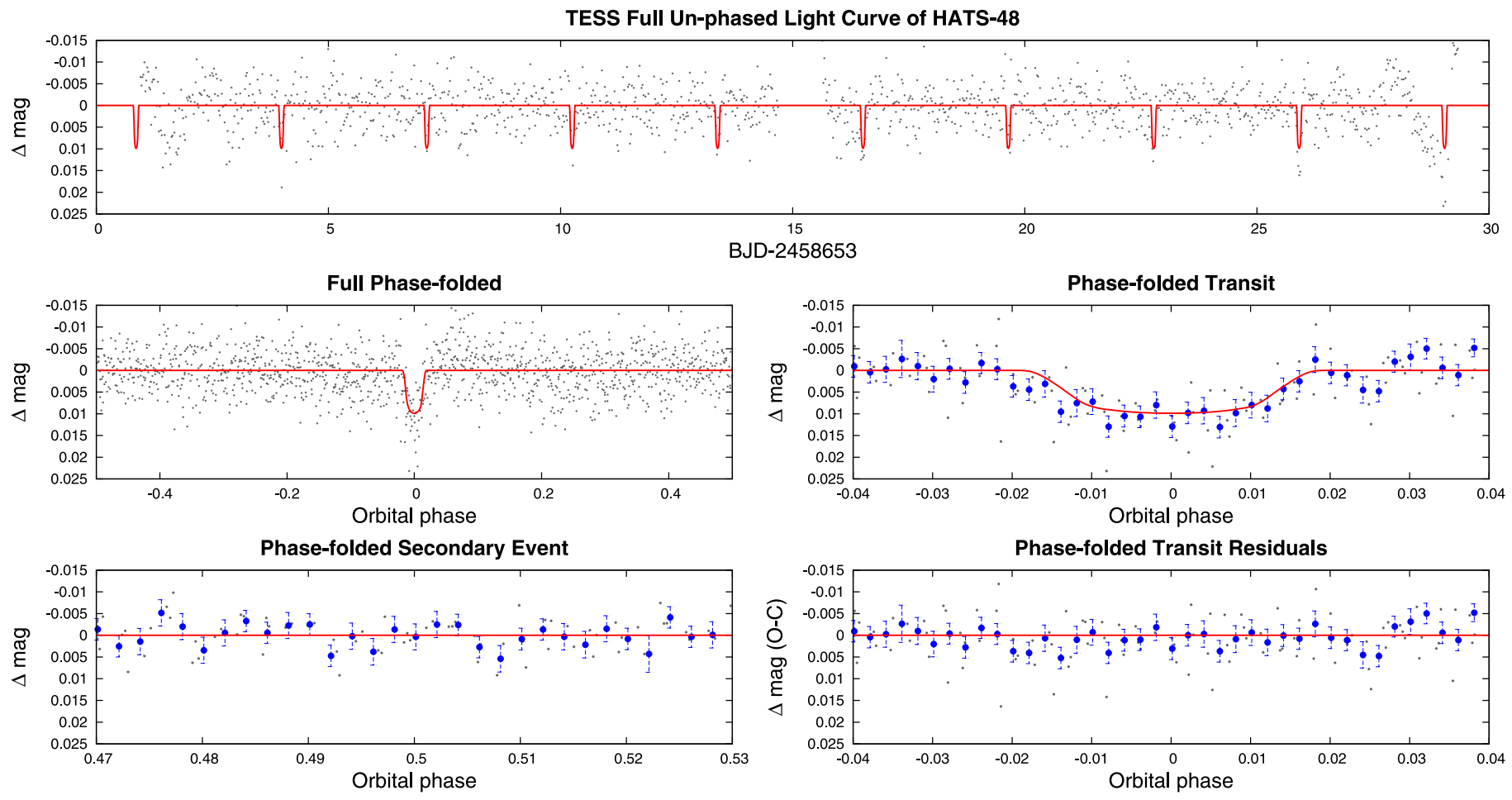

Figure 6. Similar to Figure 5, but here we show the TESS long-cadence light curve for HATS-48A. The model lines account for the 30 minute integrations. Other observations included in our analysis of this system are shown in Figure 2. 

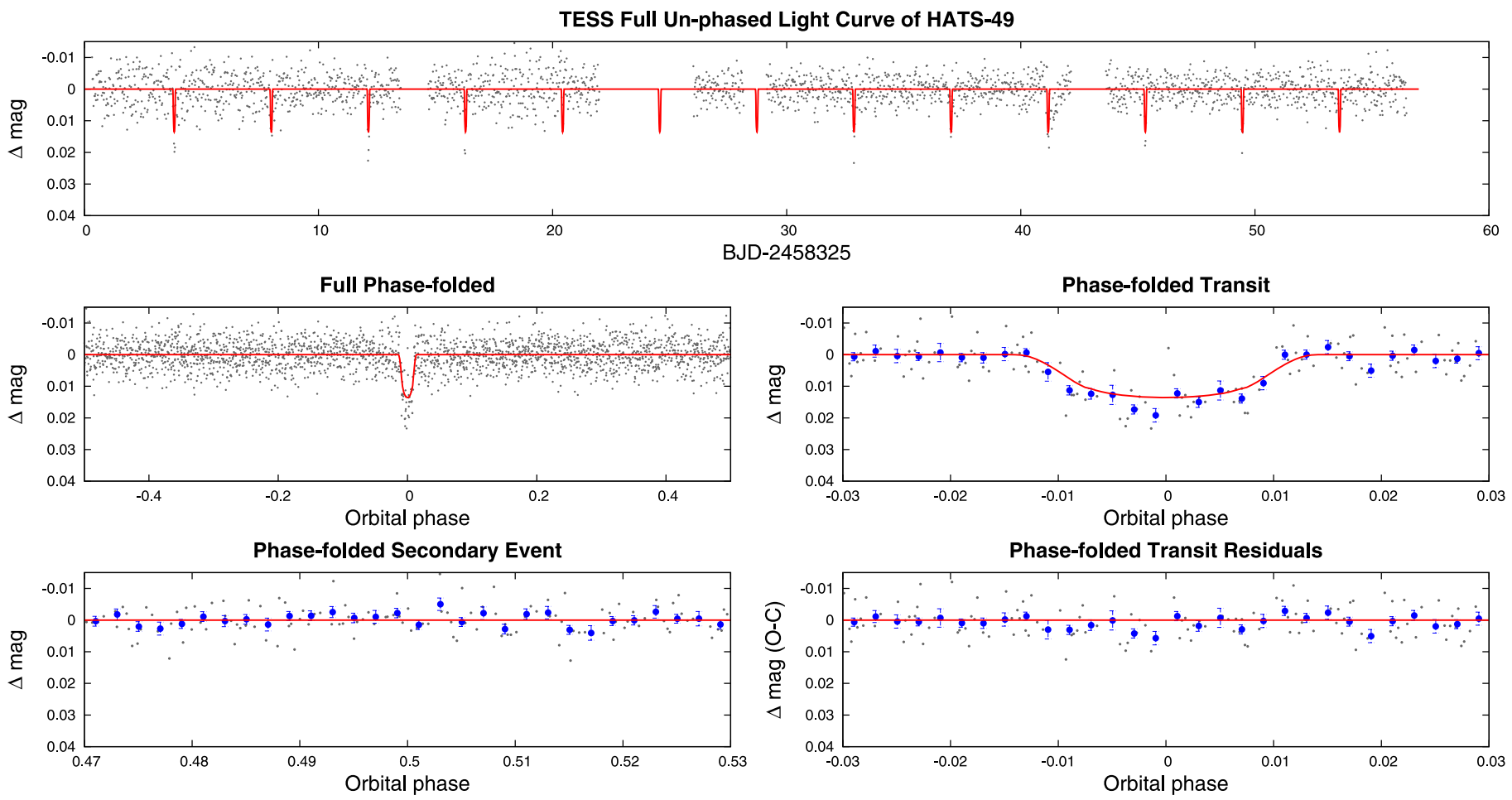

Figure 7. Similar to Figure 5, but here we show the TESS long-cadence light curve for HATS-49. Other observations included in our analysis of this system are shown in Figure 3.
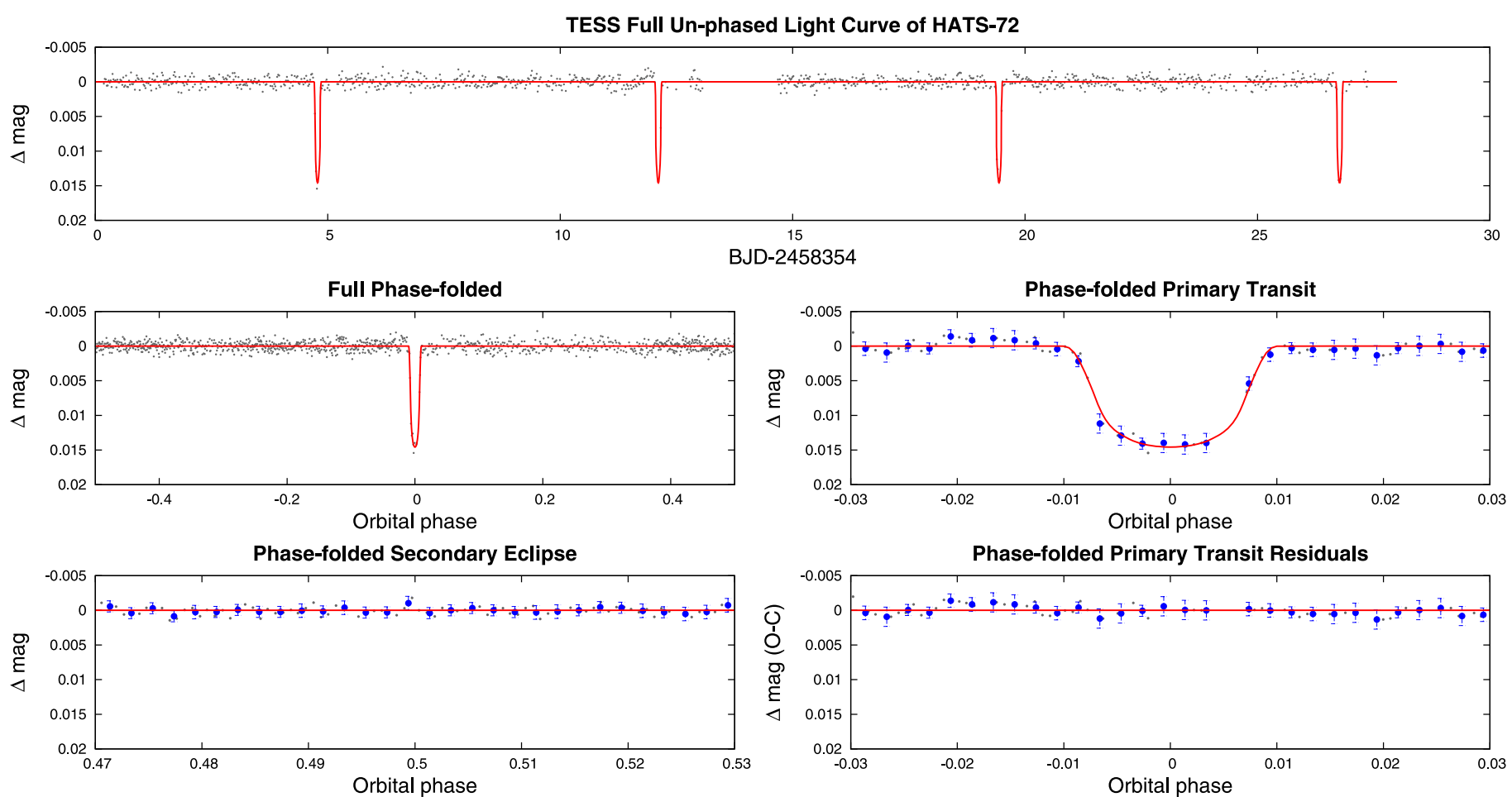

Figure 8. Similar to Figure 8, but here we show the TESS long-cadence light curve for HATS-72. Other observations included in our analysis of this system are shown in Figure 4 and 9.

of HATS-47 by fitting and subtracting a harmonic series to the data. The harmonic-filtered light curve is then used in the analysis (Section 3).

The other three targets were not included in the set of stars observed in short-cadence mode by the mission, so only $\sim 30$ minute integrations from the Full Frame Images (FFIs) are available for these objects. Of these, HATS-72 was bright enough to have a light curve produced from the FFI observations by the TESS Quick Look Pipeline (QLP; Huang et al. 2018) at MIT. We made use of the detrended light curve for HATS-72 produced by this pipeline. For HATS-48A and HATS-49, which were not processed by SPOC and were too 

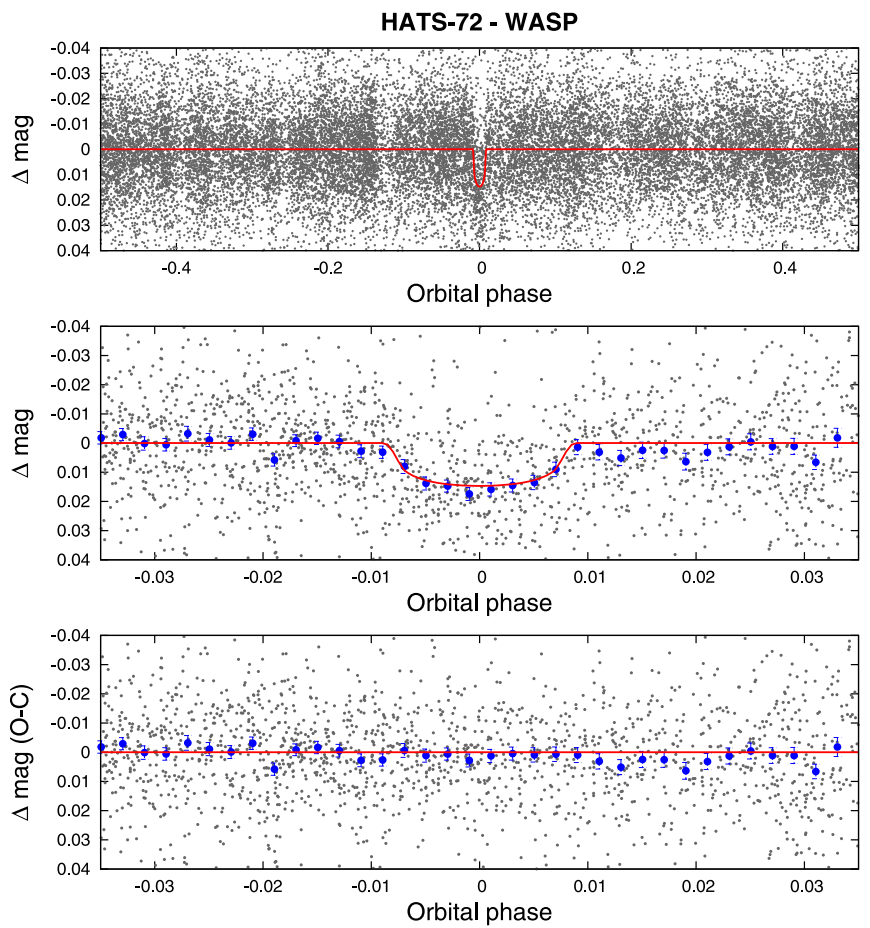

Figure 9. WASP light curve for HATS-72, displayed in a similar fashion to the HATSouth light curve shown in Figure 4 (see the caption in Figure 1). Other observations included in our analysis of this system are shown in Figure 4 and 8 .

faint to be processed through the QLP, we extracted light curves from the TESS FFIs using Lightkurve (Lightkurve Collaboration et al. 2018) and TESSCut (Brasseur et al. 2019), using a B-spline to remove trends on timescales longer than the transits, and made use of these data in our analysis.

We note that HATS-47 and HATS-48A suffer significant blending from nearby stars in the low spatial-resolution TESS images, and the Sector 13 observations also have significant scattered light.

\subsubsection{WASP}

HATS-72 was observed by WASP-South over the period 2006 May to 2012 June, accumulating 24,200 data points. WASP-South is an array of eight cameras combining 200-mm $f / 1.8$ lenses with $2 \mathrm{k} \times 2 \mathrm{k}$ CCDs and observing with a broad, 400-700 nm bandpass (Pollacco et al. 2006). It observed visible fields with a typical cadence of 15 minutes on each clear night. After identification of a candidate 7.33 day transit periodicity (see Collier Cameron et al. 2007), HATS-72 was placed on the WASP-South follow-up program in 2013 January. This led to nine RVs being observed with the Euler/CORALIE spectrograph (e.g., Triaud et al. 2013) over 2013-2018, and the observation in 2018 July of a transit with TRAPPIST-South (e.g., Gillon et al. 2013), using an $I+z$ filter. The data were compatible with the transiting object being a planet, leading to a provisional designation as WASP-191b, but the low amplitude meant that detection of orbital motion was not secure. Plans to acquire HARPS observations were then superseded by confirmation of the planet by the HATSouth team.

\subsection{Spectroscopic Observations}

The spectroscopic observations carried out to confirm and characterize the four transiting planet systems presented here are summarized in Table 3. The facilities are: WiFeS on the ANU $2.3 \mathrm{~m}$ (Dopita et al. 2007), PFS on the Magellan $6.5 \mathrm{~m}$ (Crane et al. 2006, 2008, 2010), FEROS on the MPG $2.2 \mathrm{~m}$ (Kaufer \& Pasquini 1998), HARPS on the ESO $3.6 \mathrm{~m}$ (Mayor et al. 2003), Coralie on the Euler 1.2 m (Queloz et al. 2001), and ESPRESSO on the VLT $8.2 \mathrm{~m}$ (Mégevand et al. 2014).

The WiFeS observations were obtained for HATS-47, HATS-48A, and HATS-49, and were used for reconnaissance to rule out common false positives, such as transiting very lowmass stars or eclipsing stellar binaries blended with a brighter giant star. The data were reduced following Bayliss et al. (2013). For each object, we obtained spectra at resolving power $R \equiv \Delta \lambda / \lambda \approx 3000$ to estimate the effective temperature, $\log g$ and $[\mathrm{Fe} / \mathrm{H}]$ of the star. Additional observations at $R \approx 7000$ were also obtained to search for any large-amplitude radial velocity variations at the $\sim 4 \mathrm{~km} \mathrm{~s}^{-1}$ level, which would indicate a stellar mass companion. All three systems were confirmed to be dwarf stars with RV variations below $4 \mathrm{~km} \mathrm{~s}^{-1}$.

We obtained PFS observations of all four systems. For each system, we obtained observations with an $\mathrm{I}_{2}$ cell, as well as observations without the cell. The $\mathrm{I}_{2}$-free observations were used to construct a template for measuring high-precision RVs from observations made with the cell, following the method of Butler et al. (1996). The PFS RV observations were included in the modeling that we performed for all four systems (Section 3.1). Spectral line Bisector Span (BS) measurements and their uncertainties were measured as described by Jordán et al. (2014) and Brahm et al. (2017a).

We obtained FEROS observations for HATS-47, HATS48A, and HATS-72. These were reduced to wavelengthcalibrated spectra, as well as high-precision RV and BS measurements using the CERES software package (Brahm et al. 2017a). Due to the faintness of HATS-47 and HATS48A, we found that the scatter in the FEROS RV measurements for these two systems was too high to be useful in constraining the RV orbital variation of the host star. For the much brighter host HATS-72, however, we did incorporate FEROS data into the analysis.

The HARPS observations of HATS-72 were also reduced using CERES. The RVs were of high enough precision to be included in our analysis of this system. The HARPS observations reported here were obtained by the HATSouth team. We note that a single HARPS observation of this system was also independently obtained by the WASP team. We do not include it in the analysis, however, because it was gathered and reduced in a different manner from the observations obtained by the HATSouth team.

Coralie observations of HATS-72 were carried out by the WASP team independently of the other reported spectroscopic observations of this system, which were gathered by the HATSouth team. The observations constrain the orbital variation of the host star due to the planet having a semiamplitude less than $\sim 50 \mathrm{~m} \mathrm{~s}^{-1}$. Additionally, no correlation is seen between the Coralie RV and BS measurements, supporting a planetary interpretation of the observations.

Finally, we obtained ESPRESSO observations of HATS-72 after the HARPS, PFS, and FEROS observations indicated a likely super-Neptune mass for the planet. The observations were carried out through the queue service mode between 2019 
Table 1

Summary of Photometric Observations

\begin{tabular}{|c|c|c|c|c|c|}
\hline Instrument/Field ${ }^{\mathrm{a}}$ & Date(s) & \# Images ${ }^{\mathrm{b}}$ & $\begin{array}{c}\text { Cadence }^{\mathrm{c}} \\
(\mathrm{sec})\end{array}$ & Filter & $\begin{array}{c}\text { Precision }^{\mathrm{d}} \\
(\mathrm{mmag})\end{array}$ \\
\hline \multicolumn{6}{|l|}{ HATS-47 } \\
\hline HS-1/G747 & 2013 Mar-Oct & 4231 & 287 & $r$ & 15.9 \\
\hline HS-3/G747 & 2013 Apr-Nov & 9045 & 297 & $r$ & 16.6 \\
\hline HS-4/G747 & 2013 Sep-Nov & 1467 & 297 & $r$ & 19.1 \\
\hline HS-5/G747 & 2013 Mar-Nov & 6022 & 297 & $r$ & 15.7 \\
\hline Swope $1 \mathrm{~m}$ & 2016 Aug 3 & 151 & 160 & $i$ & 1.9 \\
\hline \multicolumn{6}{|l|}{ HATS-48A } \\
\hline HS-2/G778 & 2011 May-2012 Nov & 2982 & 287 & $r$ & 14.6 \\
\hline HS-4/G778 & 2011 Jul-2012 Nov & 3726 & 298 & $r$ & 13.5 \\
\hline HS-6/G778 & 2011 Apr-2012 Oct & 2215 & 298 & $r$ & 14.3 \\
\hline TESS/Sector 13 & 2019 Jun-Jul & 1301 & 1798 & $T$ & 5.2 \\
\hline HS-4/G754 & 2012 Sep-2013 Jan & 3197 & 292 & $r$ & 17.7 \\
\hline HS-6/G754 & 2012 Sep-Dec & 3002 & 285 & $r$ & 17.0 \\
\hline HS-1/G755 & $2011 \mathrm{Jul}-2012$ Oct & 5249 & 292 & $r$ & 18.1 \\
\hline HS-3/G755 & $2011 \mathrm{Jul}-2012 \mathrm{Oct}$ & 4828 & 287 & $r$ & 19.0 \\
\hline HS-5/G755 & 2011 Jul-2012 Oct & 6024 & 296 & $r$ & 16.0 \\
\hline TESS/Sector 1 & 2018 Jul-Aug & 1078 & 1798 & $T$ & 4.8 \\
\hline TESS/Sector 2 & 2018 Aug-Sep & 1219 & 1798 & $T$ & 3.7 \\
\hline LCO $1 \mathrm{~m} /$ Sinistro & 2014 Nov 19 & 34 & 288 & $i$ & 1.5 \\
\hline LCO $1 \mathrm{~m} /$ Sinistro & 2015 Aug 31 & 37 & 223 & $i$ & 1.8 \\
\hline LCO $1 \mathrm{~m} / \mathrm{SBIG}$ & 2015 Sep 13 & 73 & 201 & $i$ & 3.9 \\
\hline LCO $1 \mathrm{~m} /$ Sinistro & 2015 Sep 17 & 30 & 223 & $i$ & 3.0 \\
\hline \multicolumn{6}{|l|}{ HATS-72 } \\
\hline
\end{tabular}

Notes.

${ }^{\text {a }}$ For HATSouth data, we list the HATSouth unit, CCD, and field name from which the observations are taken. HS-1 and -2 are located at Las Campanas Observatory in Chile, HS-3 and -4 are located at the H.E.S.S. site in Namibia, and HS-5 and -6 are located at Siding Spring Observatory in Australia. Each unit has four CCDs. Each field corresponds to one of 838 fixed pointings used to cover the full $4 \pi$ celestial sphere. All data from a given HATSouth field and CCD number are reduced together, while detrending through External Parameter Decorrelation (EPD) is done independently for each unique unit+CCD + field combination.

${ }^{\mathrm{b}}$ Excluding any outliers or other data not included in the modeling.

${ }^{\mathrm{c}}$ The median time between consecutive images rounded to the nearest second. Due to factors such as weather, the day-night cycle, guiding, and focus corrections, the cadence is only approximately uniform over short timescales.

${ }^{\mathrm{d}}$ The rms of the residuals from the best-fit model. Note that, in the case of HATSouth and TESS observations, the transit may appear artificially shallower due to overfiltering and/or blending from unresolved neighbors. As a result, the $\mathrm{S} / \mathrm{N}$ of the transit may be less than what would be calculated from $R_{p} / R_{\star}$ and the rms estimates given here.

May 11 and 2019 June 4 . We obtained seven exposures of $1800 \mathrm{~s}$, all made at an airmass below 1.2, achieving a mean $\mathrm{S} / \mathrm{N}$ per resolution element of 50 at $550 \mathrm{~nm}$. The ESPRESSO observations were reduced using version 1.3.2 of the ESPRESSO Data Analysis System (Cupani et al. 2018) in the ESO Reflex environment (Freudling et al. 2013), with the spectra cross-correlated against a K5 spectral mask to produce high-precision RVs. A more complete description of the observational setup and reduction procedure for the ESPRESSO observations obtained through our program will be provided in a forthcoming publication on the HATS-73 system (D. Bayliss et al. 2020, in preparation).

We also used the FEROS and $\mathrm{I}_{2}$-free PFS observations to determine high-precision stellar atmospheric parameters for the host stars using the ZASPE package (Brahm et al. 2017b). The parameters that we measured include the effective temperature $T_{\text {eff }}$, surface gravity $\log g$, metallicity $[\mathrm{Fe} / \mathrm{H}]$, and $v \sin i$. The method involves cross-correlating the observed spectra against synthetic model spectra, and then obtaining error estimates for the parameters by performing a bootstrap analysis where the 
Table 2

Light Curve Data for HATS-47, HATS-48A, HATS-49, and HATS-72

\begin{tabular}{|c|c|c|c|c|c|c|}
\hline Object $^{\mathrm{a}}$ & $\begin{array}{c}\text { BJD }^{\mathrm{b}} \\
(2,400,000+)\end{array}$ & $\mathrm{Mag}^{\mathrm{c}}$ & $\sigma_{\mathrm{Mag}}$ & Mag(orig) ${ }^{\mathrm{d}}$ & Filter & $\overline{\text { Instrument }}$ \\
\hline HATS-47 & 2456492.53516 & -0.01555 & 0.01509 & $\ldots$ & $r$ & HS \\
\hline HATS-47 & 2456570.99127 & -0.01117 & 0.01041 & $\ldots$ & $r$ & HS \\
\hline HATS-47 & 2456551.37754 & 0.03031 & 0.01653 & $\ldots$ & $r$ & HS \\
\hline HATS-47 & 2456500.38125 & -0.01951 & 0.01123 & $\ldots$ & $r$ & HS \\
\hline HATS-47 & 2456433.69366 & -0.00988 & 0.01094 & $\ldots$ & $r$ & HS \\
\hline HATS-47 & 2456516.07293 & -0.00670 & 0.01008 & $\ldots$ & $r$ & HS \\
\hline HATS-47 & 2456465.07680 & -0.02574 & 0.01287 & $\ldots$ & $r$ & HS \\
\hline
\end{tabular}

Notes. This table is available in a machine-readable form in the online journal. A portion is shown here for guidance regarding its form and content.

${ }^{\text {a }}$ Either HATS-47, HATS-48A, HATS-49, or HATS-72.

b Barycentric Julian Dates in this paper are reported on the Barycentric Dynamical Time (TDB) system.

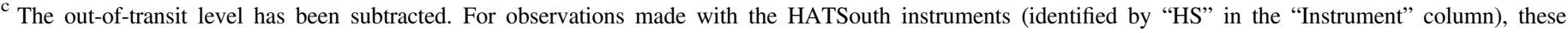

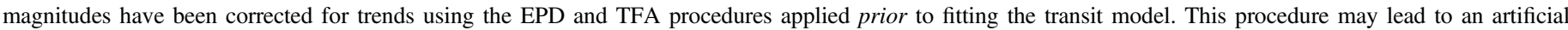

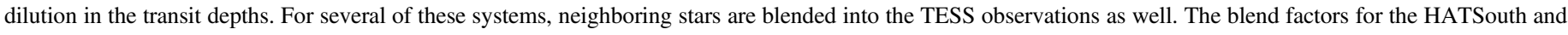

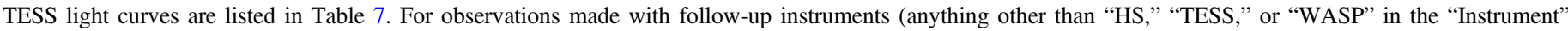

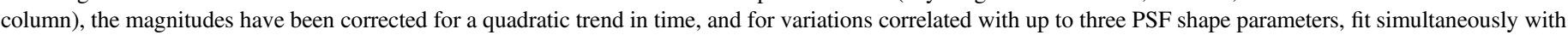

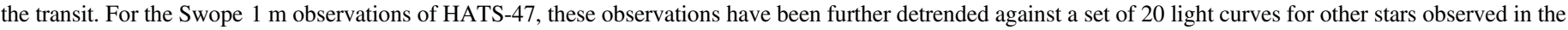
field.

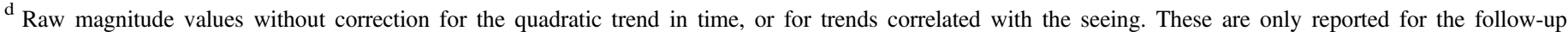
observations.

(This table is available in its entirety in machine-readable form.)

regions in the spectra that are most sensitive to changes in the atmospheric parameters are randomly adjusted based on the observed distribution of systematic mismatches between the observations and the best-matching model. This method allows for realistic parameter uncertainties that account, in a principled fashion, for systematic errors in the theoretical models. We performed this analysis on the PFS template spectra for HATS-47 and HATS-49, and on the FEROS spectra for HATS-48A and HATS-72. The resulting parameters are listed in Table 4.

The high-precision RV and BS measurements that were used in the analysis are given in Table 5 for all four systems.

\subsection{Photometric Follow-up Observations}

Follow-up higher-precision ground-based photometric transit observations were obtained for all four systems, as summarized in Table 1. The facilities used for this purpose are: the Swope $1 \mathrm{~m}$ telescope at Las Campanas Observatory in Chile, $1 \mathrm{~m}$ telescopes from the Las Cumbres Observatory (LCOGT) network (Brown et al. 2013), and the $0.6 \mathrm{~m}$ TRAPPIST-South telescope at La Silla Observatory (Gillon et al. 2013). The follow-up observations using the Swope $1 \mathrm{~m}$ and the LCOGT $1 \mathrm{~m}$ network were performed by the HATSouth team, while the TRAPPIST-South observations of HATS-72 were performed by the WASP team. The exposure time for the TRAPPISTSouth observations was $10 \mathrm{~s}$, though with read-out, the median cadence was $21 \mathrm{~s}$ as listed in Table 1.

Figure 11 shows example $40^{\prime \prime} \times 40^{\prime \prime}$ images, centered on each target, selected from our photometric follow-up observations. In each case, we overlay sources from the Gaia DR2 catalog, which is based on higher spatial resolution and deeper imaging than the photometric follow-up observations themselves. For all four objects, all known neighbors have been resolved by the groundbased photometric follow-up observations.
Observations with the Swope $1 \mathrm{~m}$ and the reduction of the data to light curves were performed as described by Penev et al. (2013). The LCOGT $1 \mathrm{~m}$ observations were carried out in a manner similar to that described by Hartman et al. (2015), but were reduced using the methods applied by Penev et al. (2013) to data from the Faulkes Telescope South $2 \mathrm{~m}$, with some updates for automation to be described by N. Espinoza et al. (2020; in preparation). The TRAPPIST-South observations were carried out and reduced as described in Gillon et al. (2013).

The time-series photometry data are available in Table 2, and are plotted in Figures 1-4.

\subsection{Search for Resolved Stellar Companions}

For HATS-47 and HATS-48A, the highest spatial resolution optical imaging available is from the Gaia mission (Gaia Collaboration et al. 2018). Gaia DR2 is sensitive to neighbors with $G \lesssim 20$ mag down to a limiting resolution of $\sim 1^{\prime \prime}$ (e.g., Ziegler et al. 2018).

There is a faint neighboring source to HATS-47 listed in the Gaia DR2 catalog at a projected separation of 6". 4 with $\Delta G=5.8 \mathrm{mag}$. This object is fully resolved by the Swope $1 \mathrm{~m}$ photometric follow-up observations for which the seeinglimited resolution ranged between 1". 4 and 2".5 FWHM. These observations show that the neighbor is not responsible for the transits, nor does it impact the transit depths measured in the follow-up observations. Based on the Gaia DR2 parallax measurements $(0.3 \pm 1.3$ mas, compared to $3.298 \pm 0.042$ mas for HATS-47), the neighboring source is in the background of HATS-47 and is not physically associated with it.

Similarly, there is a faint neighbor to HATS-48A in the Gaia DR2 catalog at a projected separation of 5!"4 and with $\Delta G=5.4 \mathrm{mag}$. The neighbor has a parallax of $3.64 \pm 0.44$ mas 

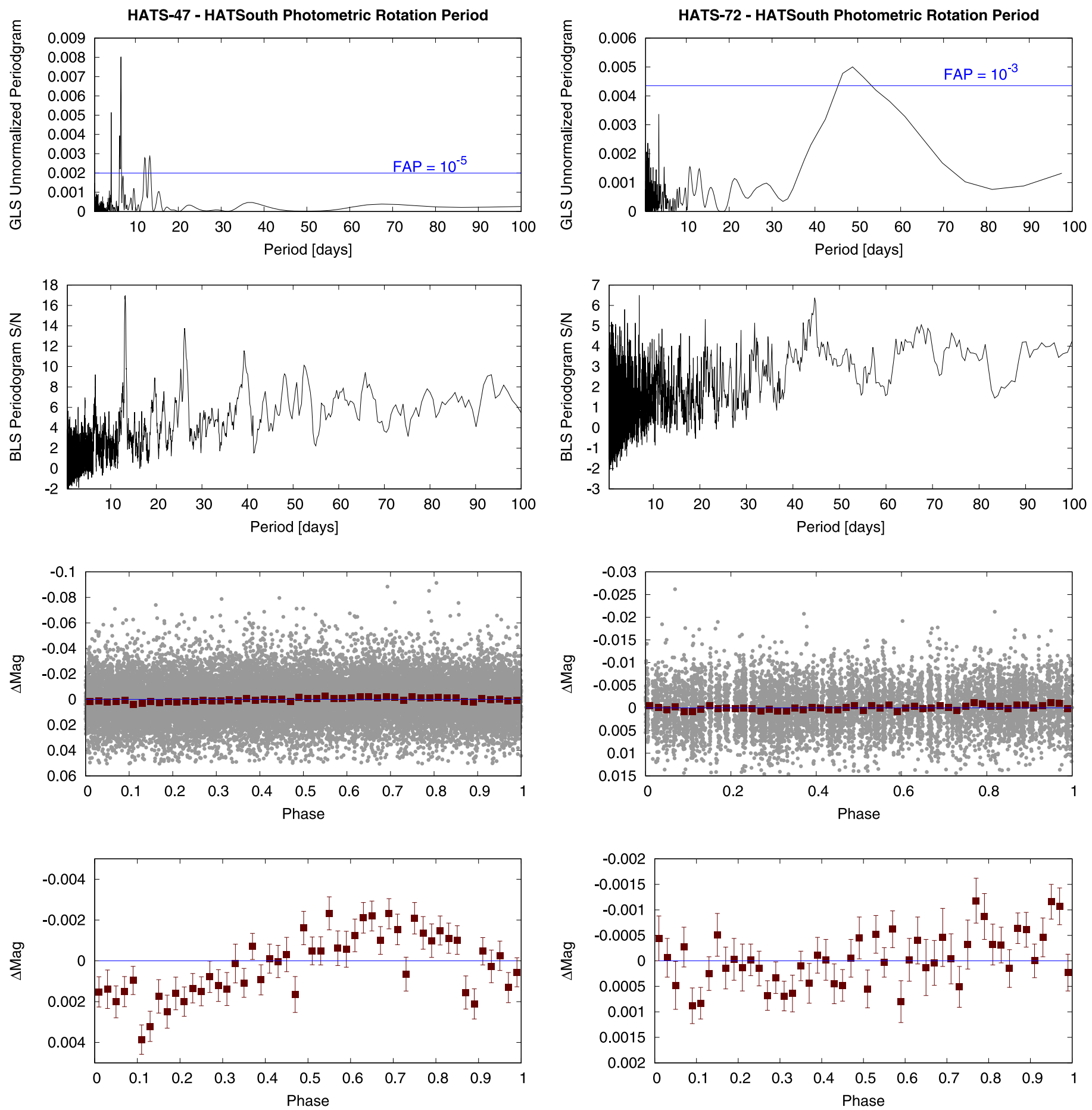

Figure 10. Detection of a strong $P=6.621581 \pm 0.000078$ day photometric rotation period signal in the HATSouth light curve of HATS-47 (left) and a tentative $P=48.725 \pm 0.015$ day signal in the HATSouth light curve of HATS-72 (right). In each case, we show the following panels. Top: Generalized Lomb-Scargle (GLS) periodogram of the combined HATSouth light curve after subtracting the best-fit transit model. Horizontal blue line shows the bootstrap-calibrated $10^{-5}$ false alarm probability level for HATS-47 and the $10^{-3}$ false alarm probability level for HATS-72. Second from top: Box-fitting Least Squares (BLS) periodogram of the same light curve. For HATS-47, there is a peak in the BLS periodogram at twice the period of the strongest peak in the GLS periodogram. For HATS-72, no significant peak is present in the BLS periodogram. Second from bottom: HATSouth light curve phase-folded at the peak GLS period. Gray points show the individual photometric measurements, while dark red filled squares show the observations binned in phase with a bin size of 0.02. Bottom: same as the second from bottom, but here we restrict the vertical range of the plot to better show the variation seen in the phase-binned measurements.

compared to $3.765 \pm 0.024$ mas for HATS-48A, and a proper motion of $\mu_{\text {R.A. }}=2.95 \pm 0.47 \mathrm{mas} \mathrm{yr}^{-1}$ and $\mu_{\text {decl. }}=5.20 \pm$

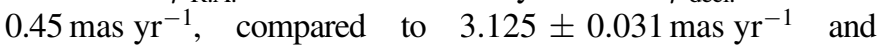
$6.146 \pm 0.029$ mas $\mathrm{yr}^{-1}$ for HATS-48A. If we assume that this source is a physical binary companion to HATS-48A, and that it is a single star, then adopting the age, mass, and metallicity for HATS-48A determined in Section 3.1 and listed in Table 6, and using the PARSEC stellar evolution models (Marigo et al. 2017), we find that $\Delta G=5.442 \pm 0.004 \mathrm{mag}$ implies a mass of $0.224 \pm 0.001 M_{\odot}$ for the companion. In that case, the predicted 
Table 3

Summary of Spectroscopy Observations

\begin{tabular}{|c|c|c|c|c|c|c|}
\hline Instrument & UT Date(s) & \# Spec. & $\begin{array}{c}\text { Res. } \\
\Delta \lambda / \lambda / 1000\end{array}$ & $\mathrm{~S} / \mathrm{N}$ Range $^{\mathrm{a}}$ & $\begin{array}{c}\gamma_{\mathrm{RV}}{ }^{\mathrm{b}} \\
\left(\mathrm{km} \mathrm{s}^{-1}\right)\end{array}$ & $\begin{array}{l}\text { RV Precision }{ }^{\mathrm{c}} \\
\quad\left(\mathrm{m} \mathrm{s}^{-1}\right)\end{array}$ \\
\hline \multicolumn{7}{|l|}{ HATS-47 } \\
\hline ANU $2.3 \mathrm{~m} / \mathrm{WiFeS}$ & 2015 Jun 1 & 1 & 3 & 28 & $\cdots$ & $\cdots$ \\
\hline ANU $2.3 \mathrm{~m} / \mathrm{WiFeS}$ & 2015 Jul 27-30 & 4 & 7 & $17-40$ & 2.0 & 4000 \\
\hline Magellan $6.5 \mathrm{~m} / \mathrm{PFS}+\mathrm{I}_{2}$ & 2016 Mar-Aug & 12 & 76 & $\cdots$ & $\cdots$ & 37 \\
\hline Magellan $6.5 \mathrm{~m} / \mathrm{PFS}$ & 2016 Mar 30 & 1 & 76 & $\cdots$ & $\cdots$ & $\cdots$ \\
\hline MPG $2.2 \mathrm{~m} /$ FEROS & 2016 Jul 1-26 & 5 & 48 & $14-33$ & 3.179 & 55 \\
\hline \multicolumn{7}{|l|}{ HATS-48A } \\
\hline ANU $2.3 \mathrm{~m} / \mathrm{WiFeS}$ & 2014 Oct 4 & 1 & 3 & 49 & $\cdots$ & $\cdots$ \\
\hline ANU $2.3 \mathrm{~m} / \mathrm{WiFeS}$ & 2014 Oct 6-8 & 2 & 7 & $52-63$ & -22.5 & 4000 \\
\hline MPG $2.2 \mathrm{~m} /$ FEROS & 2015 Jun-Oct & 10 & 48 & $17-36$ & -22.457 & 75 \\
\hline Magellan $6.5 \mathrm{~m} / \mathrm{PFS}+\mathrm{I}_{2}$ & 2015 Jun-Oct & 12 & 76 & $\cdots$ & $\cdots$ & 30 \\
\hline Magellan $6.5 \mathrm{~m} / \mathrm{PFS}$ & 2015 Jul 1 & 1 & 76 & $\cdots$ & $\cdots$ & $\cdots$ \\
\hline \multicolumn{7}{|l|}{ HATS-49 } \\
\hline ANU $2.3 \mathrm{~m} / \mathrm{WiFeS}$ & 2014 Oct $4-5$ & 3 & 3 & $24-45$ & $\cdots$ & $\cdots$ \\
\hline ANU $2.3 \mathrm{~m} / \mathrm{WiFeS}$ & 2014 Oct $5-7$ & 2 & 7 & $24-48$ & 8.1 & 4000 \\
\hline Magellan $6.5 \mathrm{~m} / \mathrm{PFS}+\mathrm{I}_{2}$ & 2015 Jan-2016 Jun & 8 & 76 & $\cdots$ & $\cdots$ & 53 \\
\hline Magellan $6.5 \mathrm{~m} / \mathrm{PFS}$ & 2015 Jan 8 & 1 & 76 & $\cdots$ & $\cdots$ & $\cdots$ \\
\hline \multicolumn{7}{|l|}{ HATS-72 } \\
\hline ESO $3.6 \mathrm{~m} / \mathrm{HARPS}$ & 2017 Apr-2018 Aug & 11 & 115 & $9-31$ & 15.955 & 12.0 \\
\hline MPG $2.2 \mathrm{~m} /$ FEROS & 2017 Jun-Aug & 6 & 48 & $24-68$ & 15.946 & 12.7 \\
\hline Euler $1.2 \mathrm{~m} /$ Coralie & 2013 Jul-2018 Jul & 9 & 60 & $42-46$ & 15.934 & 15 \\
\hline Magellan $6.5 \mathrm{~m} / \mathrm{PFS}+\mathrm{I}_{2}$ & 2018 May-Aug & 3 & 76 & $\cdots$ & $\cdots$ & 14.7 \\
\hline Magellan $6.5 \mathrm{~m} / \mathrm{PFS}$ & 2018 Jun 23 & 1 & 76 & $\cdots$ & $\cdots$ & $\cdots$ \\
\hline VLT $8.2 \mathrm{~m} /$ ESPRESSO & 2019 May-Jun & 7 & 140 & 50 & 15.997 & 10.5 \\
\hline
\end{tabular}

Notes.

a S/N per resolution element near $5180 \AA$ A. This was not measured for all of the instruments.

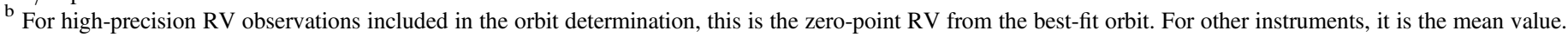
We only provide this quantity when applicable.

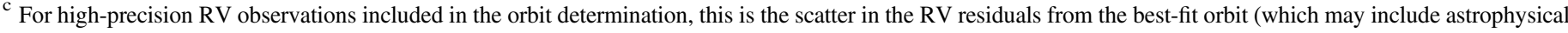

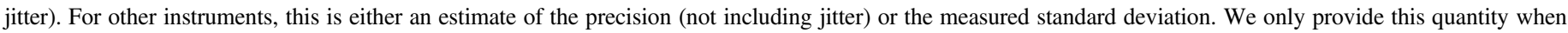
applicable.

$B P$ and $R P$ magnitude differences between the companion and HATS-48A are $\triangle B P=6.74 \mathrm{mag}$ and $\triangle R P=4.88 \mathrm{mag}$, which are comparable to the observed differences of $\triangle B P=6.11 \pm$ $0.12 \mathrm{mag}$ and $\triangle R P=4.80 \pm 0.02 \mathrm{mag}$. Note that theoretical isochrones are known to have errors in matching the optical photometry of late $\mathrm{M}$ dwarf stars, particularly in blue filters, so although the observed magnitude differences are off by more than the formal uncertainties, the results are close enough for us to conclude that the faint neighbor is most likely a bound physical companion to HATS-48A. Given the distance measured to HATS-48A, the neighbor is currently at a projected physical separation of $\sim 1400$ au from HATS-48A.

We obtained high-angular resolution imaging of HATS-49 using the Astralux Sur imager (Hippler et al. 2009) on the New Technology Telescope (NTT). Observations were carried out in $z^{\prime}$-band on the night of 2015 December 23, and were reduced as in Espinoza et al. (2016). No neighbor was detected, and we place limits of $\Delta z^{\prime}>1.8 \pm 0.2 \mathrm{mag}$ on neighbors down to 0 ." 138 , and $\Delta z^{\prime}>3.1 \pm 0.4 \mathrm{mag}$ at $\sim 1$ ". 5 . The image and contrast curve are shown in Figure 12. We also note that there is no neighbor within 10" of HATS-49 listed in Gaia DR2.

High-angular resolution imaging of HATS-72 was reported by Ziegler et al. (2020), who carried out speckle imaging with SOAR to search for resolved stellar companions to 542 TESS planet candidate hosts. They report that no companion to HATS-72 was detected, and place magnitude contrast limits of
$\Delta m>2.11 \mathrm{mag}$ and $\Delta m>3.60 \mathrm{mag}$ at separations of 0 "! 15 and $1^{\prime \prime}$, respectively. We also note that there is no neighbor within $10^{\prime \prime}$ of HATS-72 listed in Gaia DR2.

\section{Analysis}

\subsection{Transiting Planet Modelling}

We analyzed the photometric, spectroscopic, and astrometric observations of each system to determine the stellar and planetary parameters following the methods described by Hartman et al. (2019), with modifications as summarized most recently by Bakos et al. (2018).

We perform a global fit to the light curves, RV curves, spectroscopically measured stellar atmospheric parameters, catalog broadband photometry, and astrometric parallax from Gaia DR2. The fit is carried out using a modified version of the LFIT program that is included in the FITSH software package (Pál 2012). The light curves are modeled using the Mandel \& Agol (2002) semi-analytic transit model with quadratic limbdarkening. The limb-darkening coefficients are allowed to vary in the fit, using the tabulations from Claret et al. (2012, 2013) and Claret (2018) to place Gaussian prior constraints on their values, assuming a prior uncertainty of 0.2 for each coefficient. The RV curves are modeled using the appropriate relations for Keplerian orbits. We include in the model several parameters for the physical and observed properties of the host star, 
Table 4

Astrometric, Spectroscopic, and Photometric Parameters for HATS-47, HATS-48A, HATS-49, and HATS-72

\begin{tabular}{|c|c|c|c|c|c|}
\hline Parameter & $\begin{array}{l}\text { HATS-47 } \\
\text { Value }\end{array}$ & $\begin{array}{l}\text { HATS-48A } \\
\text { Value }\end{array}$ & $\begin{array}{l}\text { HATS-49 } \\
\text { Value }\end{array}$ & $\begin{array}{l}\text { HATS-72 } \\
\text { Value }\end{array}$ & Source \\
\hline \multicolumn{6}{|c|}{ Astrometric properties and cross identifications } \\
\hline 2MASS-ID... & $19095625-4939538$ & $19144126-5934458$ & $00262717-5620395$ & $22360631-1659597$ & \\
\hline TIC-ID ... & 158297421 & 201642601 & 281541545 & 188570092 & \\
\hline TOI-ID... & 1073.01 & $\ldots$ & $\ldots$ & 294.01 & \\
\hline GAIA DR2-ID... & 6658373007402886400 & 6638412919991750912 & 4919770108539385472 & 2594869603582993792 & \\
\hline R.A. $(J 2000) \ldots$ & $19^{\mathrm{h}} 09^{\mathrm{m}} 56^{\mathrm{s}} .2504$ & $19^{\mathrm{h}} 14^{\mathrm{m}} 41.2748 \mathrm{~s}$ & $00^{\mathrm{h}} 26^{\mathrm{m}} 27.1829 \mathrm{~s}$ & $22^{\mathrm{h}} 36^{\mathrm{m}} 06.3190 \mathrm{~s}$ & GAIA DR2 \\
\hline Decl. $(\mathrm{J} 2000) \ldots$ & $-49^{\circ} 39^{\prime} 53^{\prime \prime} 868$ & $-59^{\circ} 34^{\prime} 45.7571^{\prime \prime}$ & $-56^{\circ} 20^{\prime} 39.5352^{\prime \prime}$ & $-16^{\circ} 59^{\prime} 59.7882^{\prime \prime}$ & GAIA DR2 \\
\hline$\mu_{\text {R.A. }}\left(\operatorname{mas~yr}^{-1}\right)$ & $3.827 \pm 0.058$ & $3.125 \pm 0.031$ & $42.581 \pm 0.035$ & $-108.621 \pm 0.090$ & GAIADR2 \\
\hline$\mu_{\text {decl. }}\left({\left.\text { mas } \mathrm{yr}^{-1}\right)}^{-}\right.$ & $4.878 \pm 0.038$ & $6.146 \pm 0.029$ & $8.264 \pm 0.030$ & $-84.412 \pm 0.078$ & GAIA DR2 \\
\hline parallax (mas) & $3.298 \pm 0.042$ & $3.765 \pm 0.024$ & $3.054 \pm 0.022$ & $7.809 \pm 0.037$ & GAIA DR2 \\
\hline \multicolumn{6}{|c|}{ Spectroscopic properties } \\
\hline$T_{\text {eff }}(\mathrm{K}) \ldots$ & $4479 \pm 51$ & $4190 \pm 100$ & $4354 \pm 70$ & $4612 \pm 76$ & ZASPE $^{\mathrm{a}}$ \\
\hline$[\mathrm{Fe} / \mathrm{H}] \ldots$ & $-0.140 \pm 0.066$ & $0.00 \pm 0.10$ & $0.080 \pm 0.084$ & $-0.040 \pm 0.050$ & ZASPE \\
\hline$v \sin i\left(\mathrm{~km} \mathrm{~s}^{-1}\right) \ldots$ & $2.47 \pm 0.70$ & $0.73 \pm 0.55$ & $0.50 \pm 0.78$ & $0.8 \pm 1.3$ & ZASPE \\
\hline$v_{\text {mac }}\left(\mathrm{km} \mathrm{s}^{-1}\right) \ldots$ & $1.994 \pm 0.077$ & $1.55 \pm 0.15$ & $1.80 \pm 0.11$ & $2.20 \pm 0.12$ & Assumed \\
\hline$v_{\text {mic }}\left(\mathrm{km} \mathrm{s}^{-1}\right) \ldots$ & $0.326 \pm 0.048$ & $0.000 \pm 0.085$ & $0.197 \pm 0.076$ & $0.443 \pm 0.061$ & Assumed \\
\hline$\gamma_{\mathrm{RV}}\left(\mathrm{m} \mathrm{s}^{-1}\right) \ldots$ & $3197 \pm 31$ & $-22460 \pm 21$ & $8100 \pm 1500$ & $15954.8 \pm 1.8$ & FEROS or WiFeS ${ }^{b}$ \\
\hline \multicolumn{6}{|l|}{ Photometric properties } \\
\hline$P_{\text {rot }}(\mathrm{d})^{\mathrm{c}}$ & $6.42 \pm 0.28$ & $\ldots$ & $\ldots$ & $48.725 \pm 0.015$ & HATSouth \\
\hline$G(\mathrm{mag})^{\mathrm{d}}$.. & $14.39980 \pm 0.00040$ & $13.89510 \pm 0.00020$ & $14.54490 \pm 0.00030$ & $12.07250 \pm 0.00030$ & GAIA DR2 \\
\hline$B P(\mathrm{mag})^{\mathrm{d}}$. & $15.0858 \pm 0.0021$ & $14.5801 \pm 0.0016$ & $15.2886 \pm 0.0022$ & $12.7084 \pm 0.0018$ & GAIA DR2 \\
\hline$R P(\mathrm{mag})^{\mathrm{d}} \ldots$ & $13.61140 \pm 0.00090$ & $13.11260 \pm 0.00090$ & $13.7214 \pm 0.0013$ & $11.3341 \pm 0.0010$ & GAIA DR2 \\
\hline$B(\operatorname{mag}) \ldots$ & $16.101 \pm 0.040$ & $15.577 \pm 0.050$ & $16.378 \pm 0.040$ & $13.572 \pm 0.030$ & APASS $^{\mathrm{e}}$ \\
\hline$V(\operatorname{mag}) \ldots$ & $14.829 \pm 0.010$ & $14.35 \pm 0.11$ & $14.998 \pm 0.040$ & $12.469 \pm 0.010$ & $\mathrm{APASS}^{\mathrm{e}}$ \\
\hline$g(\mathrm{mag}) \ldots$ & $15.480 \pm 0.010$ & $14.935 \pm 0.030$ & $15.668 \pm 0.040$ & $12.995 \pm 0.010$ & APASS $^{\mathrm{e}}$ \\
\hline$r(\mathrm{mag}) \ldots$ & $14.398 \pm 0.010$ & $13.821 \pm 0.050$ & $14.496 \pm 0.010$ & $11.998 \pm 0.010$ & $\mathrm{APASS}^{\mathrm{e}}$ \\
\hline$i(\mathrm{mag}) \ldots$ & $14.009 \pm 0.010$ & $13.69 \pm 0.17$ & $14.14 \pm 0.12$ & $11.622 \pm 0.030$ & APASS $^{\mathrm{e}}$ \\
\hline$J(\mathrm{mag}) \ldots$ & $12.653 \pm 0.023$ & $12.160 \pm 0.024$ & $12.692 \pm 0.024$ & $10.424 \pm 0.023$ & 2MASS \\
\hline$H(\mathrm{mag}) \ldots$ & $12.026 \pm 0.023$ & $11.591 \pm 0.026$ & $12.105 \pm 0.024$ & $9.907 \pm 0.026$ & 2MASS \\
\hline$K_{s}(\mathrm{mag}) \ldots$ & $11.926 \pm 0.025$ & $11.427 \pm 0.021$ & $11.938 \pm 0.023$ & $9.764 \pm 0.021$ & 2MASS \\
\hline$W 1(\mathrm{mag}) \ldots$ & $11.867 \pm 0.023$ & $11.364 \pm 0.023$ & $11.903 \pm 0.023$ & $9.687 \pm 0.024$ & WISE \\
\hline$W 2(\mathrm{mag}) \ldots$ & $11.947 \pm 0.024$ & $11.458 \pm 0.021$ & $11.990 \pm 0.022$ & $9.772 \pm 0.020$ & WISE \\
\hline W3 (mag)... & $11.707 \pm 0.192$ & $11.433 \pm 0.135$ & $12.212 \pm 0.256$ & $9.675 \pm 0.043$ & WISE \\
\hline
\end{tabular}

Notes.

${ }^{\mathrm{a}}$ ZASPE $=$ Zonal Atmospherical Stellar Parameter Estimator routine for the analysis of high-resolution spectra (Brahm et al. 2017b), applied to the PFS spectra of HATS-47 and HATS-49, and to the FEROS spectra of HATS-48A and HATS-72.

${ }^{\mathrm{b}}$ The error on $\gamma_{\mathrm{RV}}$ is determined from the orbital fit to the RV measurements, and does not include the systematic uncertainty in transforming the velocities to the IAU standard system. The velocities have not been corrected for gravitational redshifts. We report the value from FEROS for HATS-47, HATS-48A, and HATS-72. For HATS-49, we report the value from WiFeS.

${ }^{\mathrm{c}}$ Photometric rotation period.

d The listed uncertainties for the Gaia DR2 photometry are taken from the catalog. For the analysis, we assume additional systematic uncertainties of 0.002 mag, $0.005 \mathrm{mag}$, and $0.003 \mathrm{mag}$ for the $G, B P$, and $R P$ bands, respectively.

${ }^{\mathrm{e}}$ From APASS DR6 as listed in the UCAC 4 catalog (Zacharias et al. 2013).

including the effective photospheric temperature, the metallicity, the distance modulus, and the $V$-band extinction $A_{V}$. These parameters are, in turn, constrained by the observed spectroscopic stellar atmospheric parameters (as measured in Section 2.2), the catalog photometry, and the parallax. Together with the parameters used to describe the transit and RV observations, these parameters are sufficient to determine the bulk physical properties of the stars and their transiting planets. We fit the data using two different methods for relating the stellar mass to the stellar radius, metallicity, and luminosity: (1) an empirical method that uses the stellar bulk density measured from the transit and RV observations to determine the stellar mass from the stellar radius, which is itself inferred from the effective temperature and luminosity (this method is similar to that of, e.g., Stassun et al. 2017), and (2) using the
PARSEC theoretical stellar evolution models (Marigo et al. 2017) to impose an additional constraint on the stellar relations that is typically tighter than the observed constraint on the stellar bulk density.

In each case, we model the data assuming the orbital eccentricity is zero, and we separately try allowing the eccentricity to be a free parameter.

A Differential Evolution Markov chain Monte Carlo method is used to explore the parameter space and estimate the uncertainties based on the posterior parameter distribution. See Hartman et al. (2019) for a full list of the parameters that we vary, as well as their assumed priors.

We include in the fit the optical broadband photometry from Gaia DR2 and APASS, NIR photometry from 2MASS, and IR photometry from Wide-field Infrared Survey Explorer (WISE). 
Table 5

Relative Radial Velocities and Bisector Spans for HATS-47, HATS-48A, HATS-49, and HATS-72

\begin{tabular}{|c|c|c|c|c|c|c|c|}
\hline System & $\begin{array}{c}\text { BJD } \\
(2,450,000+)\end{array}$ & $\begin{array}{c}\mathrm{RV}^{\mathrm{a}} \\
\left(\mathrm{m} \mathrm{s}^{-1}\right)\end{array}$ & $\begin{array}{l}\sigma_{\mathrm{RV}}^{\mathrm{b}} \\
\left(\mathrm{m} \mathrm{s}^{-1}\right)\end{array}$ & $\begin{array}{c}\mathrm{BS} \\
\left(\mathrm{m} \mathrm{s}^{-1}\right)\end{array}$ & $\begin{array}{c}\sigma_{\mathrm{BS}} \\
\left(\mathrm{m} \mathrm{s}^{-1}\right)\end{array}$ & Phase & Instrument \\
\hline HATS-47 & 7472.87421 & -73.25 & 9.58 & -379.5 & 95.6 & 0.408 & PFS \\
\hline HATS-47 & 7474.88339 & 17.75 & 9.26 & 84.1 & 58.3 & 0.920 & PFS \\
\hline HATS-47 & 7477.90415 & 68.00 & 12.60 & $\ldots$ & $\ldots$ & 0.690 & PFS \\
\hline HATS-47 & 7507.88072 & -33.23 & 11.94 & -331.8 & 71.1 & 0.332 & PFS \\
\hline HATS-47 & 7530.84061 & -93.65 & 13.98 & 60.7 & 177.1 & 0.185 & PFS \\
\hline HATS-47 & 7558.79646 & 43.22 & 10.65 & 89.5 & 133.0 & 0.311 & PFS \\
\hline HATS-47 & 7615.65549 & 29.50 & 8.55 & -98.7 & 49.9 & 0.806 & PFS \\
\hline
\end{tabular}

Notes. This table is available in a machine-readable form in the online journal. A portion is shown here for guidance regarding its form and content.

a The zero-point of these velocities is arbitrary. An overall offset $\gamma_{\text {rel }}$ fitted independently to the velocities from each instrument has been subtracted.

${ }^{\mathrm{b}}$ Internal errors, excluding the component of astrophysical jitter allowed to vary in the fit.

For WISE, we exclude the W4 band for all systems, as none of the objects were detected in that bandpass, while for HATS-47, HATS-48A, and HATS-49, we also exclude the W3 band, as the photometric uncertainty exceeds $0.1 \mathrm{mag}$ in this bandpass for these three objects. These observations, together with the stellar atmospheric parameters, the parallax, and the reddening, constrain the luminosity of the star. To model the reddening, we assume an $R_{V}=3.1$ Cardelli et al. (1989) dust law parameterized by $A_{V}$, and use the MWDUST 3D Galactic extinction model (Bovy et al. 2016) to place a prior constraint on its value.

We find that, for all four transiting planet systems, the orbits are consistent with being circular when the eccentricities are varied, and that the stellar parameters are more robustly constrained when imposing the theoretical stellar evolution model constraints. We therefore choose to adopt the parameters that stem from fixing the orbit to be circular and imposing the stellar evolution models as a constraint on the stellar physical parameters.

The best-fit models are compared to the various observational data for the four transiting planet systems in Figures 1-9. The adopted stellar parameters derived from the analysis are listed in Table 6, while the adopted planetary parameters are listed in Table 7 . We also list in Table 7 the $95 \%$ confidence upper limit on the eccentricity that comes from allowing the eccentricity to vary in the fit.

\subsection{Stellar Blend Modelling}

We also performed a blend modeling of each system following Hartman et al. (2019), where we attempt to fit all of the observations (except the RV data) using various combinations of stars, with parameters constrained by the PARSEC models. For all four objects, we find that a model consisting of a single star with a transiting planet provides a better fit (a greater likelihood and a lower $\chi^{2}$ ) to the light curves, spectroscopic stellar atmospheric parameters, broadband catalog photometry, and astrometric parallax measurements than the best-fit blended stellar eclipsing binary models. The blended stellar eclipsing binary models involve more free parameters than the transiting planet model, and thus can be rejected on the grounds that they are both poorer-fitting and more complicated models. Moreover, the fact that Keplerian orbital variations consistent with transiting planets are observed for all four objects, and that large spectral line bisector span variations are not observed, is further evidence in favor of the transiting planet interpretation of the observations.

We also attempted to fit the systems as unresolved stellar binaries with a planet transiting the brighter stellar component. For HATS-47 and HATS-48A, we find that there is no significant improvement in $\chi^{2}$ when adding an unresolved stellar binary companion compared to the model of a single star with a transiting planet. For HATS-49 and HATS-72, adding an unresolved companion does improve the fit, with $\Delta \chi^{2}=$ -17.4 for HATS-49, and $\Delta \chi^{2}=-39.8$ for HATS-72. At face value, this may be taken as evidence for an unresolved stellar companion to both of these objects. For HATS-49, this modeling yields a mass of $0.245 \pm 0.039 M_{\odot}$ for the unresolved stellar companion, while for HATS-72, we find $0.317 \pm 0.025 M_{\odot}$. However, there is no other independent evidence for a stellar companion (such as a long-term trend in the RVs) for either of these objects. Furthermore, some lowmass stars (including late $\mathrm{K}$ dwarfs) have been observed to have larger radii than predicted by theoretical isochrones (e.g., Torres 2013). If this is the case for either HATS-49 or HATS-72, invoking an additional star would appear to reconcile the observations to the model-leading to a better fit but an erroneous conclusion. Therefore, we do not consider this to be a clear detection of a stellar companion for either HATS-49 or HATS-72. Instead, we present in Table 6 the 95\% confidence upper limits on the mass of an unresolved companion for all four objects. High angular resolution imaging, long-term RV observations, and/or Gaia astrometry could potentially detect the companions if they are present.

\section{Discussion}

We have presented the discovery of four transiting giant planets on close-in orbits around $\mathrm{K}$ dwarf stars. Three of the planets presented in this paper, HATS-47b, HATS-48Ab, and HATS49b, are comparable in mass to Saturn. The masses are $0.369_{-0.021}^{+0.031} M_{\mathrm{J}}, 0.243_{-0.030}^{+0.022} M_{\mathrm{J}}$, and $0.353_{-0.027}^{+0.038} M_{\mathrm{J}}$, respectively. Despite their relatively short orbital periods of 3.9228 days, 3.1317 days, and 4.1480 days, all three of these planets can be considered warm giants with predicted equilibrium temperatures of $852.9 \pm 4.7 \mathrm{~K}, 954.6 \pm 4.8 \mathrm{~K}$, and $834.8 \pm 3.6 \mathrm{~K}$ (estimated assuming zero-albedo and full redistribution of heat; note that the small uncertainties listed here do not account for the possibility 

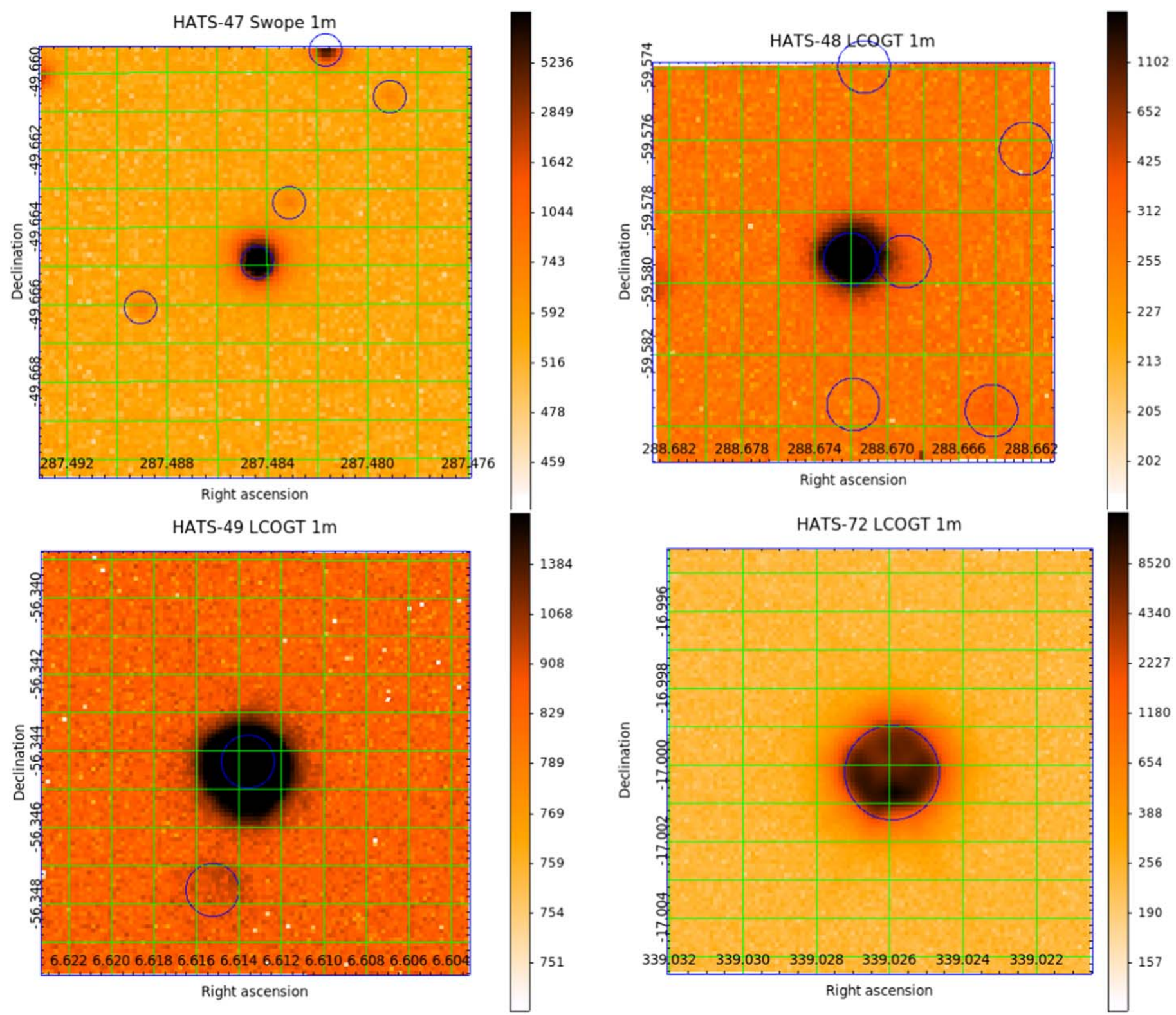

Figure 11. Snapshot $40^{\prime \prime} \times 40^{\prime \prime}$ images of HATS-47, HATS-48A, HATS-49, and HATS-72 selected from the ground-based time-series photometric follow-up observations obtained for each object. Each image is centered on the transiting planet host star. The blue circles indicate the positions of sources in the Gaia DR2 catalog, which is based on higher spatial resolution and deeper observations than the images shown here. The radius of each circle is equal to the approximate HWHM of the image PSF. Note that the LCOGT $1 \mathrm{~m}$ observations were carried out with defocusing to improve the photometric precision, while the Swope $1 \mathrm{~m}$ observations were carried out in-focus with the resolution limited by atmospheric seeing. The color scale indicates the number of counts (in ADU) in each calibrated image pixel, and is shown on an inverted logarithmic scale. All known neighbors are resolved in the ground-based photometric follow-up observations carried out for each system.

that these assumptions are wrong, nor for systematic errors in the stellar evolution models). This is due to the planets orbiting cool K dwarf stars with respective masses of $0.674_{-0.012}^{+0.016} M_{\odot}$, $0.7279 \pm 0.0066 M_{\odot}$, and $0.7133 \pm 0.0075 M_{\odot}$. The fourth planet, HATS-72b, is a super-Neptune with a mass of $0.1254 \pm 0.0039 M_{\mathrm{J}}$, and with a somewhat longer orbital period of 7.3279 days. This planet also orbits a cool $\mathrm{K}$ dwarf star of mass $0.7311 \pm 0.0028 M_{\odot}$, and has a modest predicted equilibrium temperature of $739.3 \pm 1.6 \mathrm{~K}$.

Figures 13 and 14 compare the planet masses, planet radii, average incident fluxes, host star masses, and orbital semimajor axes of the four systems presented in this paper to other published giant transiting planets listed in the NASA Exoplanet Archive with $R_{p}>0.5 R_{\mathrm{J}}$ and with measured masses. We show the comparison to all planets that satisfy these restrictions, and to only those found around stars with $M<0.8 M_{\odot}$. The four objects presented here are consistent with established trends. Notably, all four objects have relatively small radii $\left(1.117 \pm 0.014 R_{\mathrm{J}}\right.$, $0.800 \pm 0.015 R_{\mathrm{J}}, 0.765 \pm 0.013 R_{\mathrm{J}}$, and $0.7224 \pm 0.0032 R_{\mathrm{J}}$, for HATS-47b-HATS-49b, and HATS-72b, respectively) as expected for their low masses and modest irradiation. This makes the planets potentially useful objects for comparing to theoretical models of giant planet structure to infer their bulk heavy element contents. The planets all have semimajor axes that are beyond the empirical minimum semimajor axis as a function of planet mass, as seen in the top-right panel of Figure 14. As seen in the bottom panels of Figures 13 and 14, the four planets discovered here are among a still fairly small sample of giant planets known around 

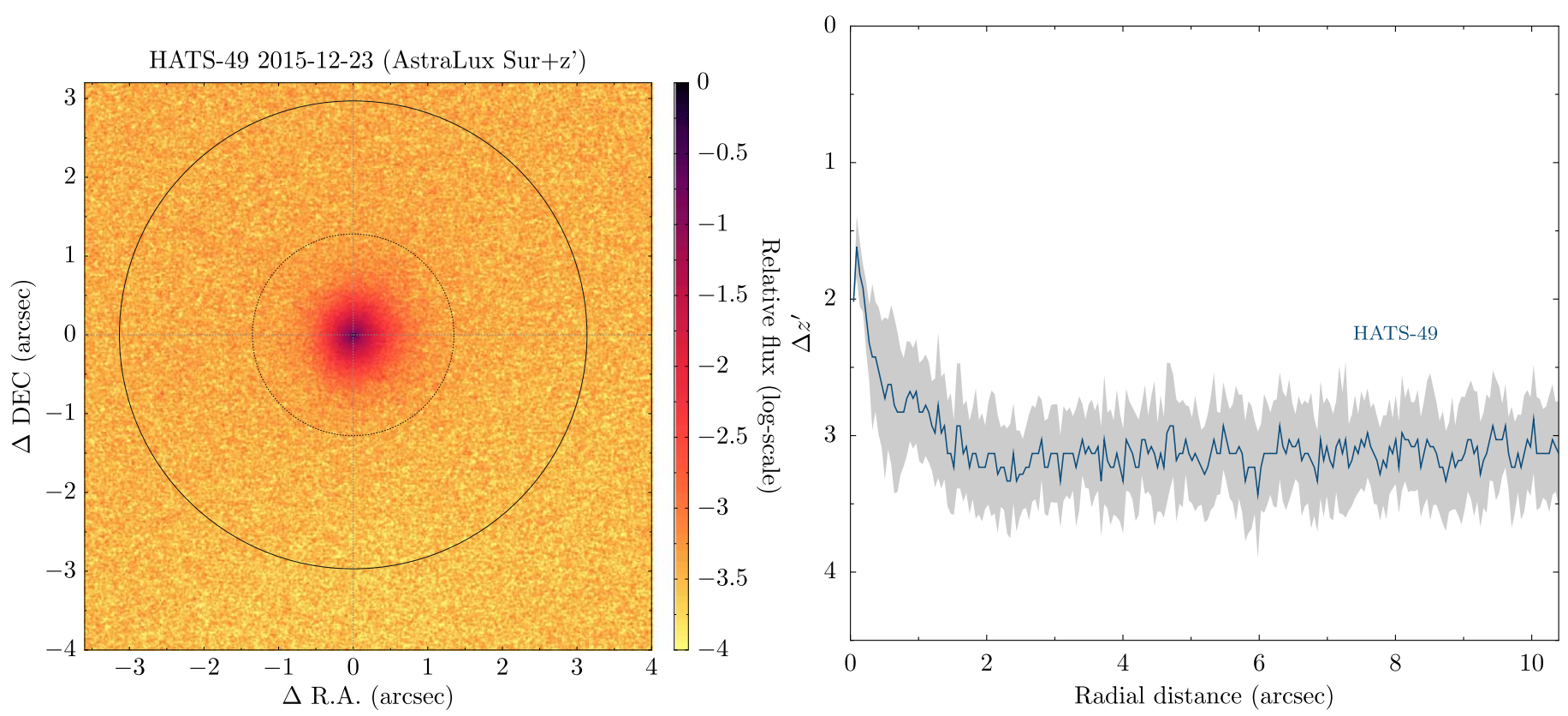

Figure 12. (left) High-resolution image of HATS-49 obtained with AstraLux Sur on the NTT through the $z^{\prime}$ filter. No neighboring sources are detected. (right) The $5 \sigma$ upper limit on the magnitude contrast of any resolved neighbor to HATS-49 based on the Astralux Sur image. Gray band shows the variation in the limit with position angle.

Table 6

Adopted Derived Stellar Parameters for HATS-47, HATS-48A, HATS-49, and HATS-72

\begin{tabular}{|c|c|c|c|c|}
\hline Parameter & $\begin{array}{c}\text { HATS-47 } \\
\text { Value }\end{array}$ & $\begin{array}{c}\text { HATS-48A } \\
\text { Value }\end{array}$ & $\begin{array}{c}\text { HATS-49 } \\
\text { Value }\end{array}$ & $\begin{array}{c}\text { HATS-72 } \\
\text { Value }\end{array}$ \\
\hline$M_{\star}\left(M_{\odot}\right) \ldots$ & $0.674_{-0.012}^{+0.016}$ & $0.7279 \pm 0.0066$ & $0.7133 \pm 0.0075$ & $0.7311 \pm 0.0028$ \\
\hline$R_{\star}\left(R_{\odot}\right) \ldots$ & $0.6564 \pm 0.0055$ & $0.7152 \pm 0.0038$ & $0.6977 \pm 0.0055$ & $0.7214 \pm 0.0021$ \\
\hline $\log g_{\star}(\mathrm{cgs}) \ldots$ & $4.633 \pm 0.011$ & $4.5909 \pm 0.0039$ & $4.6036 \pm 0.0077$ & $4.5853 \pm 0.0021$ \\
\hline$\rho_{\star}\left(\mathrm{g} \mathrm{cm}^{-3}\right) \ldots$ & $3.360_{-0.099}^{+0.130}$ & $2.804 \pm 0.036$ & $2.961 \pm 0.073$ & $2.743 \pm 0.020$ \\
\hline$L_{\star}\left(L_{\odot}\right) \ldots$ & $0.1599 \pm 0.0031$ & $0.1955 \pm 0.0042$ & $0.1641 \pm 0.0026$ & $0.2193 \pm 0.0021$ \\
\hline$T_{\text {eff } \star}(\mathrm{K}) \ldots$ & $4512 \pm 19$ & $4546_{-18}^{+23}$ & $4405 \pm 15$ & $4656.1 \pm 8.9$ \\
\hline$[\mathrm{Fe} / \mathrm{H}] \ldots$ & $-0.113 \pm 0.035$ & $0.186 \pm 0.051$ & $0.208 \pm 0.053$ & $0.099 \pm 0.014$ \\
\hline Age $(\mathrm{Gyr}) \ldots$ & $8.1_{-4.3}^{+2.9}$ & $11.97_{-0.61}^{+0.42}$ & $10.5_{-2.0}^{+1.4}$ & $12.17_{-0.45}^{+0.24}$ \\
\hline$A_{V}(\operatorname{mag}) \ldots$ & $0.108 \pm 0.032$ & $0.112 \pm 0.033$ & $0.046 \pm 0.014$ & $0.0270 \pm 0.0080$ \\
\hline Distance $(\mathrm{pc}) \ldots$ & $301.7 \pm 1.9$ & $265.4 \pm 1.7$ & $324.6 \pm 2.2$ & $127.66 \pm 0.52$ \\
\hline $\mathrm{M}_{\mathrm{B}}\left(M_{\odot}\right)^{\mathrm{a}}$ & $<0.23$ & 0.22 & $<0.31$ & $<0.36$ \\
\hline
\end{tabular}

Notes. The listed parameters are those determined through the joint differential evolution Markov Chain analysis described in Section 3.1. For all four systems, the RV observations are consistent with a circular orbit, and we assume a fixed circular orbit in generating the parameters listed here. Systematic errors in the bolometric correction tables or stellar evolution models are not included, and may dominate the error budget for some of these parameters.

${ }^{a}$ For HATS-47, HATS-49, and HATS-72, we list the 95\% confidence upper limit on the mass of any unresolved stellar companion based on modeling the system as a blend between a transiting planet system and an unresolved wide stellar binary companion (Section 3.2). For HATS-48A, we list the estimated mass for the 5."4 neighbor in Gaia DR2 that we determined to be a common-proper-motion and common-parallax companion to HATS-48A (Section 2.4).

stars with $M<0.8 M_{\odot}$, and may be useful in that sense for studying the formation and properties of close-in giant planets around low-mass stars.

HATS-47b and HATS-72b are also notable for their relatively deep transits. With a transit depth of 3\%, (e.g., Figure 1), HATS47 is among the deepest known transiting planet systems. Only HATS-71b (Bakos et al. 2018), WTS-2b (Birkby et al. 2014), HATS-6b (Hartman et al. 2015), and Kepler-45b (Johnson et al. 2012) are known to have deeper transits. Nearly as deep are the transits of WASP-80b (Triaud et al. 2013), POTS-1b (Koppenhoefer et al. 2013), Qatar-2b (Bryan et al. 2012), and CoRoT-2b (Alonso et al. 2008). The large transit depth makes HATS-47b a potentially attractive target for follow-up observations, such as transmission spectroscopy, for which the signal strength scales with the transit depth. With a transit depth of $1.1 \%$ (e.g., Figure 4), HATS-72b stands out as having the deepest transits among all known planets with $M_{p}<0.15 M_{\mathrm{J}}$, making it a valuable target as well for transmission spectroscopy, in this case to study the atmosphere of a super-Neptune. With an optical magnitude of $V=12.469 \pm 0.010 \mathrm{mag}$ and NIR magnitude of $J=10.424 \pm 0.023 \mathrm{mag}$, HATS-72 is only slightly fainter than HAT-P-26 $(V=11.74 \mathrm{mag}, J=10.08 \mathrm{mag})$, which hosts a Neptune. HATS-72 is also significantly brighter than the superEarth-hosting $\mathrm{K} 2-18$ in the visual band $(V=13.5)$, and only somewhat fainter at near-infrared wavelengths than K2-18 $(J=9.76 \mathrm{mag})$. Both of these planets produce shallower transits 
Table 7

Adopted Orbital and Planetary Parameters for HATS-47b, HATS-48Ab, HATS-49b, and HATS-72b

\begin{tabular}{|c|c|c|c|c|}
\hline Parameter & $\begin{array}{c}\text { HATS-47b } \\
\text { Value }\end{array}$ & $\begin{array}{c}\text { HATS-48Ab } \\
\text { Value }\end{array}$ & $\begin{array}{c}\text { HATS-49b } \\
\text { Value }\end{array}$ & $\begin{array}{l}\text { HATS-72b } \\
\text { Value }\end{array}$ \\
\hline \multicolumn{5}{|l|}{ Light-curve parameters } \\
\hline$P$ (days) $\ldots$ & $3.9228038 \pm 0.0000022$ & $3.1316666 \pm 0.0000037$ & $4.1480467 \pm 0.0000037$ & $7.3279474 \pm 0.0000016$ \\
\hline$T_{c}\left(\mathrm{BJD} \_\mathrm{TDB}\right)^{\mathrm{a}} \ldots$ & $2457365.35804 \pm 0.00029$ & $2457100.55022 \pm 0.00045$ & $2457105.16480 \pm 0.00054$ & $2458087.647820 \pm 0.000075$ \\
\hline$T_{14}(\text { days })^{\mathrm{a}} \ldots$ & $0.08343 \pm 0.00089$ & $0.09774 \pm 0.00050$ & $0.09775 \pm 0.00095$ & $0.12853 \pm 0.00030$ \\
\hline$T_{12}=T_{34}(\text { days })^{\mathrm{a}} \ldots$ & $0.02313 \pm 0.00067$ & $0.01015 \pm 0.00020$ & $0.01184 \pm 0.00036$ & $0.012258 \pm 0.000080$ \\
\hline$a / R_{\star} \ldots$ & $13.98 \pm 0.15$ & $11.330 \pm 0.049$ & $13.91 \pm 0.11$ & $19.821 \pm 0.048$ \\
\hline$\zeta / R_{\star}{ }^{\mathrm{b}} \ldots$ & $31.93 \pm 0.46$ & $22.825_{-0.098}^{+0.130}$ & $23.22 \pm 0.31$ & $17.195 \pm 0.049$ \\
\hline$R_{p} / R_{\star} \ldots$ & $0.1746 \pm 0.0014$ & $0.1148 \pm 0.0020$ & $0.1127 \pm 0.0015$ & $0.10290 \pm 0.00034$ \\
\hline$b^{2} \ldots$ & $0.508_{-0.014}^{+0.014}$ & $0.0069_{-0.0046}^{+0.0080}$ & $0.176_{-0.028}^{+0.022}$ & $0.0233_{-0.0066}^{+0.0054}$ \\
\hline$b \equiv a \cos i / R_{\star} \ldots$ & $0.7127_{-0.0096}^{+0.0096}$ & $0.083_{-0.035}^{+0.039}$ & $0.420_{-0.035}^{+0.025}$ & $0.153_{-0.023}^{+0.017}$ \\
\hline$i(\operatorname{deg}) \ldots$ & $87.080 \pm 0.061$ & $89.58 \pm 0.18$ & $88.27_{-0.11}^{+0.16}$ & $89.560_{-0.050}^{+0.070}$ \\
\hline \multicolumn{5}{|l|}{ Dilution factors ${ }^{\mathrm{c}}$} \\
\hline HATSouth $1 \ldots$ & $0.920 \pm 0.037$ & $0.998940 \pm 0.000094$ & $0.811 \pm 0.049$ & $0.868 \pm 0.022$ \\
\hline HATSouth $2 \ldots$ & $\ldots$ & $\ldots$ & $0.799 \pm 0.049$ & $\ldots$ \\
\hline TESS ... & $0.869 \pm 0.012$ & $0.597 \pm 0.024$ & $0.755 \pm 0.069$ & $0.97763 \pm 0.00052$ \\
\hline WASP ... & $\ldots$ & $\ldots$ & $\ldots$ & $0.984 \pm 0.012$ \\
\hline \multicolumn{5}{|l|}{ Limb-darkening coefficients ${ }^{\mathrm{d}}$} \\
\hline$c_{1}, r \ldots$ & $0.49 \pm 0.13$ & $0.36 \pm 0.14$ & $0.67 \pm 0.12$ & $0.423_{-0.080}^{+0.061}$ \\
\hline$c_{2}, r \ldots$ & $0.28_{-0.12}^{+0.15}$ & $0.32 \pm 0.15$ & $0.16 \pm 0.17$ & $0.508 \pm 0.088$ \\
\hline$c_{1}, i \ldots$ & $0.53 \pm 0.13$ & $0.22 \pm 0.11$ & $0.34 \pm 0.11$ & $0.316 \pm 0.059$ \\
\hline$c_{2}, i \ldots$ & $0.38_{-0.16}^{+0.12}$ & $0.17 \pm 0.16$ & $0.06 \pm 0.14$ & $0.444 \pm 0.097$ \\
\hline$c_{1}, T \ldots$ & $0.460 \pm 0.100$ & $0.41 \pm 0.12$ & $0.56 \pm 0.17$ & $0.503 \pm 0.060$ \\
\hline$c_{2}, T \ldots$ & $0.41 \pm 0.11$ & $0.34 \pm 0.14$ & $0.24 \pm 0.15$ & $0.403 \pm 0.084$ \\
\hline$c_{1}, I+z \ldots$ & $\cdots$ & $\ldots$ & $\ldots$ & $0.262 \pm 0.060$ \\
\hline$c_{2}, I+z \ldots$ & $\ldots$ & $\ldots$ & $\cdots$ & $0.305 \pm 0.078$ \\
\hline$c_{1}$, WASP $\ldots$ & $\cdots$ & $\cdots$ & $\cdots$ & $0.524 \pm 0.077$ \\
\hline$c_{2}$, WASP $\ldots$ & $\cdots$ & $\cdots$ & $\cdots$ & $0.349 \pm 0.074$ \\
\hline \multicolumn{5}{|l|}{ RV parameters } \\
\hline$K\left(\mathrm{~m} \mathrm{~s}^{-1}\right) \ldots$ & $61.7 \pm 4.3$ & $41.8 \pm 4.4$ & $55.9 \pm 5.5$ & $16.15 \pm 0.51$ \\
\hline$e^{\mathrm{e}} \ldots$ & $<0.088$ & $<0.162$ & $<0.071$ & $<0.013$ \\
\hline RV jitter PFS $\left(\mathrm{m} \mathrm{s}^{-1}\right) \ldots$ & $40.4 \pm 8.9$ & $25.2 \pm 6.2$ & $57 \pm 13$ & $6.4 \pm 1.7$ \\
\hline RV jitter ESPRESSO $\left(\mathrm{m} \mathrm{s}^{-1}\right) \ldots$ & $\ldots$ & $\ldots$ & $\ldots$ & $8.25 \pm 0.32$ \\
\hline RV jitter HARPS $\left(\mathrm{m} \mathrm{s}^{-1}\right) \ldots$ & $\ldots$ & $\ldots$ & $\ldots$ & $1.13 \pm 0.42$ \\
\hline RV jitter FEROS $\left(\mathrm{m} \mathrm{s}^{-1}\right) \ldots$ & $\ldots$ & $\ldots$ & $\ldots$ & $18.7 \pm 6.3$ \\
\hline \multicolumn{5}{|l|}{ Planetary parameters } \\
\hline$M_{p}\left(M_{\mathrm{J}}\right) \ldots$ & $0.369_{-0.021}^{+0.031}$ & $0.243_{-0.030}^{+0.022}$ & $0.353_{-0.027}^{+0.038}$ & $0.1254 \pm 0.0039$ \\
\hline$R_{p}\left(R_{\mathrm{J}}\right) \ldots$ & $1.117 \pm 0.014$ & $0.800 \pm 0.015$ & $0.765 \pm 0.013$ & $0.7224 \pm 0.0032$ \\
\hline$C\left(M_{p}, R_{p}\right)^{\mathrm{g}} \ldots$ & -0.00 & 0.16 & 0.31 & 0.30 \\
\hline$\rho_{p}\left(\mathrm{~g} \mathrm{~cm}^{-3}\right) \ldots$ & $0.331 \pm 0.027$ & $0.589 \pm 0.067$ & $0.986 \pm 0.094$ & $0.4110_{-0.0100}^{+0.0150}$ \\
\hline $\log g_{p}(\mathrm{cgs}) \ldots$ & $2.866 \pm 0.032$ & $2.978_{-0.058}^{+0.036}$ & $3.177 \pm 0.040$ & $2.774 \pm 0.013$ \\
\hline$a(\mathrm{AU}) \ldots$ & $0.04269_{-0.00025}^{+0.00033}$ & $0.03769 \pm 0.00011$ & $0.04515 \pm 0.00016$ & $0.066517 \pm 0.000085$ \\
\hline$T_{\mathrm{eq}}(\mathrm{K}) \ldots$ & $852.9 \pm 4.7$ & $954.6 \pm 4.8$ & $834.8 \pm 3.6$ & $739.3 \pm 1.6$ \\
\hline$\Theta^{h} \ldots$ & $0.0418_{-0.0025}^{+0.0034}$ & $0.0317_{-0.0041}^{+0.0027}$ & $0.0585_{-0.0045}^{+0.0059}$ & $0.03150 \pm 0.00097$ \\
\hline $\log _{10}\langle F\rangle(\operatorname{cgs})^{\mathrm{i}} \ldots$ & $8.0770 \pm 0.0095$ & $8.2725 \pm 0.0086$ & $8.0394 \pm 0.0073$ & $7.8292 \pm 0.0038$ \\
\hline
\end{tabular}

Notes. For all systems, we adopt a model in which the orbit is assumed to be circular. See the discussion in Section 3.1 .

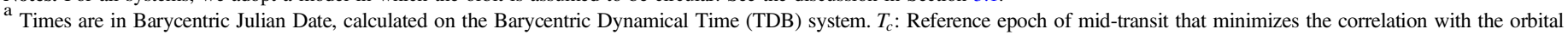
period. $T_{12}$ : total transit duration, time between first to last contact; $T_{12}=T_{34}$ : ingress/egress time, time between first and second or between third and fourth contact.

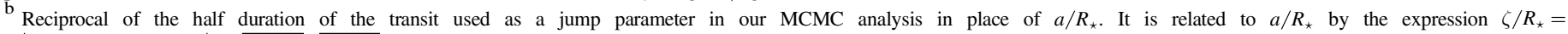
$a / R_{\star}(2 \pi(1+e \sin \omega)) /\left(P \sqrt{1-b^{2}} \sqrt{1-e^{2}}\right.$ ) (Bakos et al. 2010).

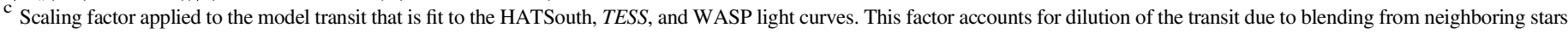

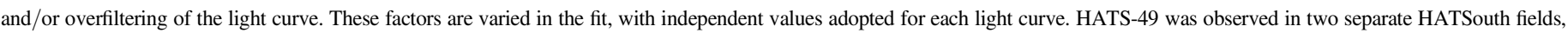
and we list the two independent dilution factors fitted for the light curves from each of these fields.

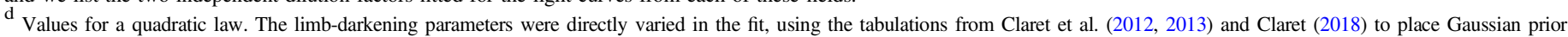
constraints on their values, assuming a prior uncertainty of 0.2 for each coefficient.

The $95 \%$ confidence upper limit on the eccentricity determined when $\sqrt{e} \cos \omega$ and $\sqrt{e} \sin \omega$ are allowed to vary in the fit.

${ }^{\mathrm{f}}$ Term added in quadrature to the formal RV uncertainties for each instrument. This is treated as a free parameter in the fitting routine.

g Correlation coefficient between the planetary mass $M_{p}$ and radius $R_{p}$ estimated from the posterior parameter distribution.

$\mathrm{h}$ The Safronov number is given by $\Theta=\frac{1}{2}\left(V_{\text {esc }} / V_{\text {orb }}\right)^{2}=\left(a / R_{p}\right)\left(M_{p} / M_{\star}\right)$ (see Hansen $\&$ Barman 2007).

${ }^{\mathrm{i}}$ Incoming flux per unit surface area, averaged over the orbit.

(This table is available in its entirety in machine-readable form.) 

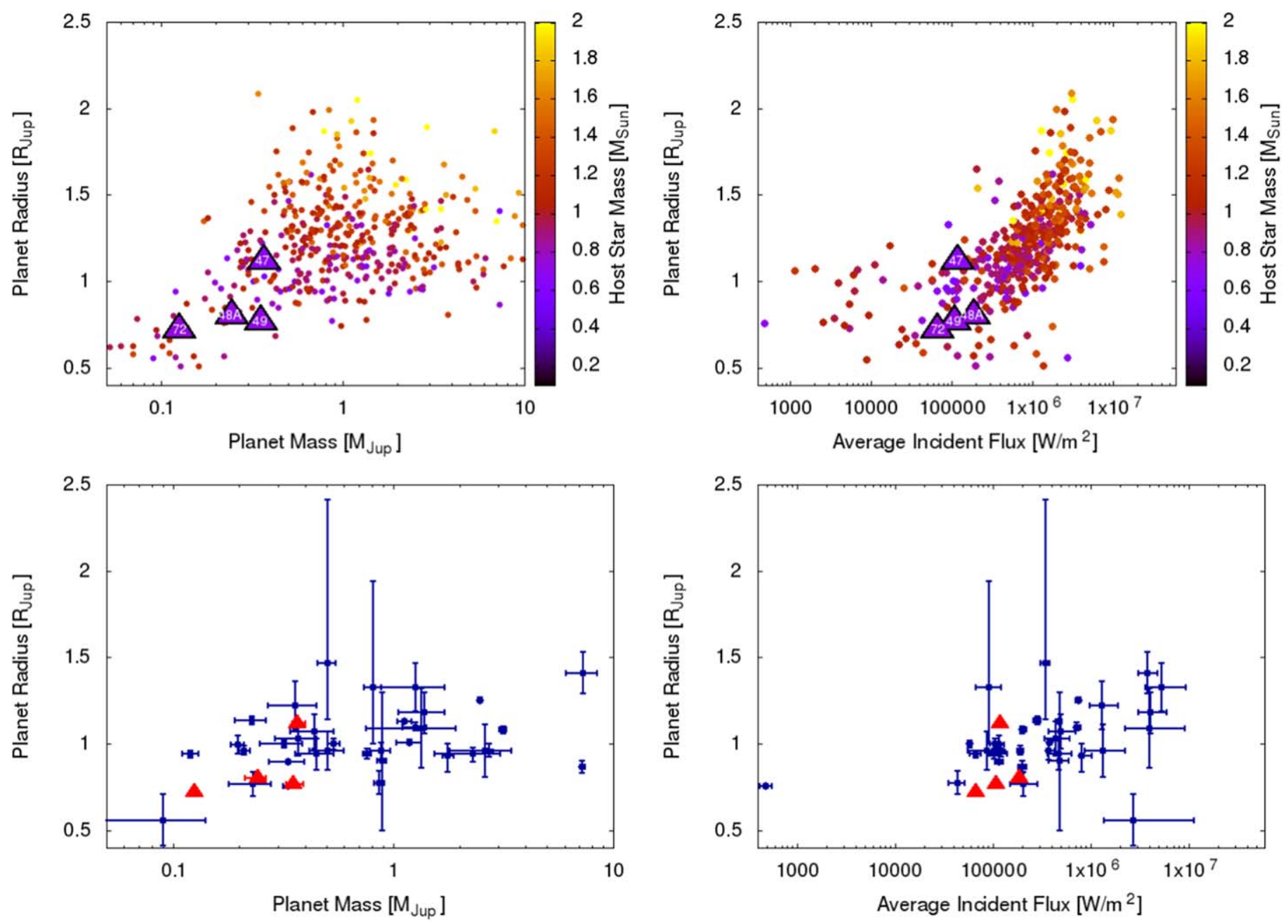

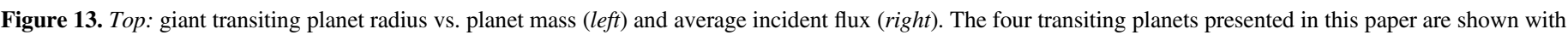

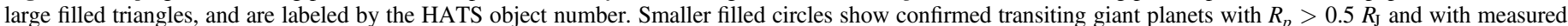

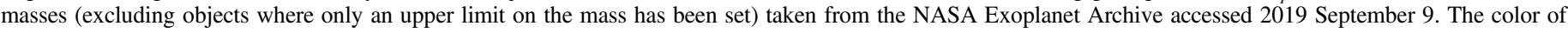

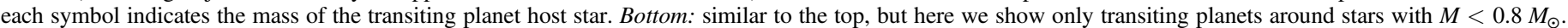

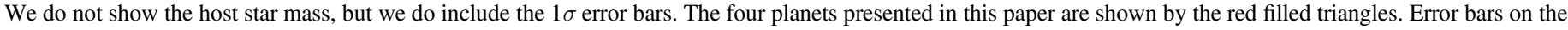
planet radius and incident flux are smaller than the symbols for these four planets.

than HATS-72b, and have had molecules detected in their atmospheres via transmission spectroscopy (Wakeford et al. 2017; Benneke et al. 2019; Tsiaras et al. 2019).

HATS-72 and HATS-73 (D. Bayliss et al.2020, in preparation) are the first two systems confirmed by our team using ESPRESSO. This facility has been vital in confirming a relatively low-amplitude signal $\left(16.15 \pm 0.51 \mathrm{~m} \mathrm{~s}^{-1}\right)$ around cool stars. The benefits of this facility are derived not only from the increased mirror size and spectrograph efficiency, but also from the redder wavelength coverage of ESPRESSO, which is important as we push to cooler host stars.

The combination of transit survey and follow-up data from three separate projects (HATSouth, TESS, and WASP) also demonstrates the benefits of collaboration between surveys going forward. This is particularly so for HATS-72b, which was independently detected by all three surveys. As TESS continues its survey of the sky for transiting planets around bright stars, most (if not all) systems that have previously been identified by ground-based surveys will be observed by TESS. Through the coordination of TFOP, redundant follow-up observations can be avoided for transiting planet systems that have already been identified and confirmed by ground-based surveys, but have not yet been published. Coordination by TFOP also ensures greater efficiency in the analysis and publication of transiting planets such as these, by enabling data independently collected by different groups to be combined and analyzed in a single work.

Finally, this paper also illustrates a useful science contribution of ground-based transit surveys that is complimentary to the primary TESS mission. Neither of the planets HATS-48Ab nor HATS-49b were identified as transiting planet candidates by the TESS team. Both of these objects are relatively faint, with $V=14.35 \pm 0.11$ mag for HATS-48A and $V=14.998 \pm 0.040$ mag for HATS49, and are not among the targets that have been searched for transits by the TESS team, which is focused on searching bright stars around which small planets may be detectable. However, to discover transiting giant planets around low-mass stars, it is necessary to search a large number of $\mathrm{M}$ dwarfs and late-K dwarfs, and thus to consider stars that are faint in the optical bandpass. Ground-based surveys like HATSouth, combined with independent analyses of the TESS FFIs being released to the public, are providing valuable contributions addressing this science topic. 

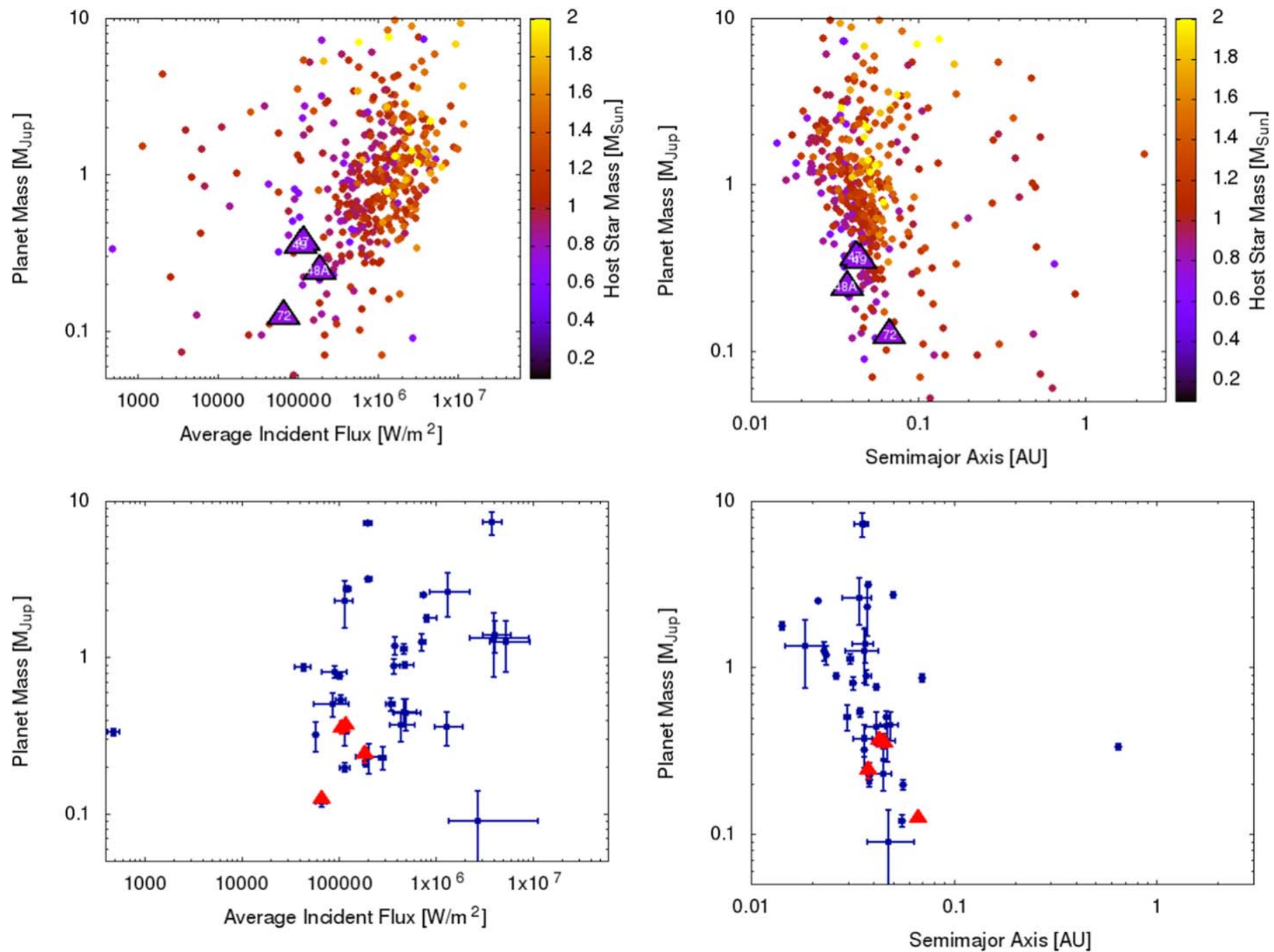

Figure 14. Similar to Figure 13, here we show giant transiting planet mass vs. average incident flux (left) and semimajor axis (right). HATS-47b and HATS-49b overlap on these plots. As in Figure 13, only planets with $R_{p}>0.5 R_{\mathrm{J}}$ and with measured masses are shown.

We thank the anonymous referee for valuable feedback, which has improved the quality of this paper. Development of the HATSouth project was funded by NSF MRI grant NSF/AST0723074, operations have been supported by NASA grants NNX09AB29G, NNX12AH91H, and NNX17AB61G, and follow-up observations have received partial support from grant NSF/AST-1108686. A.J. acknowledges support from FONDECYT project 1171208 and by the Ministry for the Economy, Development, and Tourism's Programa Iniciativa Científica Milenio through grant IC 120009, awarded to the Millennium Institute of Astrophysics (MAS). L.M. acknowledges support from the Italian Ministry of Instruction, University, and Research (MIUR) through FFABR 2017 fund. L.M. acknowledges support from the University of Rome Tor Vergata through "Mission: Sustainability 2016" fund. K.P. acknowledges support from NASA ATP grant 80NSSC18K1009. V.S. acknowledges support from BASAL CATA PFB-06. J.N.W. thanks the Heising-Simons foundation for support. I.J.M.C. acknowledges support from the NSF through grant AST-1824644, and from NASA through Caltech/JPL grant RSA-1610091. Support for this work was provided to J.K.T. by NASA through Hubble Fellowship grant HST-HF2-51399.001 awarded by the Space Telescope Science Institute, which is operated by the Association of Universities for
Research in Astronomy, Inc., for NASA, under contract NAS526555. This work is based on observations made with ESO Telescopes at the La Silla Observatory. This paper also makes use of observations from the LCOGT network. Some of this time was awarded by NOAO. We acknowledge the use of the AAVSO Photometric All-Sky Survey (APASS), funded by the Robert Martin Ayers Sciences Fund, and the SIMBAD database, operated at CDS, Strasbourg, France. Operations at the MPG $2.2 \mathrm{~m}$ Telescope are jointly performed by the Max Planck Gesellschaft and the European Southern Observatory. We thank the MPG $2.2 \mathrm{~m}$ telescope support team for their technical assistance during observations. TRAPPIST-South is a project funded by the Belgian F.R.S.-FNRS under grant FRFC 2.5.594.09.F, with the participation of the Swiss FNS. The research leading to these results has received funding from the ARC grant for Concerted Research Actions, financed by the Wallonia-Brussels Federation. E.J. and M.G. are F.R.S.-FNRS Senior Research Associates. Contributions at the University of Geneva by L.N., M.L., and S.U. were carried out within the framework of the National Centre for Competence in Research "PlanetS" supported by the Swiss National Science Foundation (SNSF). M.L. acknowledges support from the Austrian Research Promotion Agency (FFG) under project 859724 "GRAPPA." This work has made use of data from the 
European Space Agency (ESA) mission Gaia (https://www. cosmos.esa.int/gaia), processed by the Gaia Data Processing and Analysis Consortium (DPAC, https://www.cosmos.esa.int/web/ gaia/dpac/consortium). Funding for the DPAC has been provided by national institutions, in particular the institutions participating in the Gaia Multilateral Agreement. This research has made use of the NASA Exoplanet Archive, which is operated by the California Institute of Technology, under contract with the National Aeronautics and Space Administration under the Exoplanet Exploration Program. This research has made use NASA's Astrophysics Data System Bibliographic Services.

Facilities: HATSouth, TESS, SuperWASP, Swope, LCOGT, TRAPPIST, Max Planck:2.2 m (FEROS), ESO:3.6 m (HARPS), Euler1.2 m (Coralie), ATT (WiFeS), Magellan:Clay (PFS), VLT (ESPRESSO), NTT (Astralux Sur), SOAR, Gaia, Exoplanet Archive.

Software: FITSH (Pál 2012), BLS (Kovács et al. 2002), VARTOOLS (Hartman \& Bakos 2016), CERES (Brahm et al. 2017a), ZASPE (Brahm et al. 2017b), SPEX-tool (Cushing et al. 2004; Vacca et al. 2004), SExtractor (Bertin \& Arnouts 1996), Astrometry.net (Lang et al. 2010), MWDUST (Bovy et al. 2016), TESSCut (Brasseur et al. 2019), Lightkurve (Lightkurve Collaboration et al. 2018), Astropy (Astropy Collaboration et al. 2013; Price-Whelan et al. 2018).

\section{ORCID iDs}

J. D. Hartman 주 https://orcid.org/0000-0001-8732-6166 Andrés Jordán (1) https://orcid.org/0000-0002-5389-3944 D. Bayliss (i) https://orcid.org/0000-0001-6023-1335 G. Á. Bakos (1) https://orcid.org/0000-0001-7204-6727 W. Bhatti $\odot$ https://orcid.org/0000-0002-0628-0088 R. Brahm (i) https://orcid.org/0000-0002-9158-7315 N. Espinoza (1) https://orcid.org/0000-0001-9513-1449 L. Mancini (1) https://orcid.org/0000-0002-9428-8732 K. Penev (1) https://orcid.org/0000-0003-4464-1371 M. Rabus (i) https://orcid.org/0000-0003-2935-7196 P. Sarkis (ib https://orcid.org/0000-0001-8128-3126 V. Suc (1) https://orcid.org/0000-0001-7070-3842 M. de Val-Borro (1) https://orcid.org/0000-0002-0455-9384

G. Zhou (1) https://orcid.org/0000-0002-4891-3517

J. D. Crane (1) https://orcid.org/0000-0002-5226-787X

R. P. Butler (16) https://orcid.org/0000-0003-1305-3761

D. R. Anderson (1) https://orcid.org/0000-0001-7416-7522

C. Hellier 나 https://orcid.org/0000-0002-3439-1439

F. J. Pozuelos (1) https://orcid.org/0000-0003-1572-7707

M. Gillon (1) https://orcid.org/0000-0003-1462-7739

M. Lendl (1) https://orcid.org/0000-0001-9699-1459

S. Udry (1) https://orcid.org/0000-0001-7576-6236

Roland Vanderspek (1) https://orcid.org/0000-0001-6763-6562

David W. Latham (ํ) https://orcid.org/0000-0001-9911-7388

Joshua N. Winn (1) https://orcid.org/0000-0002-4265-047X

Jessie Christiansen (i) https://orcid.org/0000-0002-8035-4778

Jon M. Jenkins (i) https://orcid.org/0000-0002-4715-9460

Jeffrey C. Smith 나 https://orcid.org/0000-0002-6148-7903

\section{References}

Alonso, R., Auvergne, M., Baglin, A., et al. 2008, A\&A, 482, L21 Astropy Collaboration, Robitaille, T. P., Tollerud, E. J., et al. 2013, A\&A, 558, A33

Bakos, G., Noyes, R. W., Kovács, G., et al. 2004, PASP, 116, 266 Bakos, G. Á, Bayliss, D., Bento, J., et al. 2018, arXiv:1812.09406
Bakos, G. Á, Csubry, Z., Penev, K., et al. 2013, PASP, 125, 154 Bakos, G. Á, Torres, G., Pál, A., et al. 2010, ApJ, 710, 1724 Bayliss, D., Zhou, G., Penev, K., et al. 2013, AJ, 146, 113 Benneke, B., Wong, I., Piaulet, C., et al. 2019, ApJL, 887, L14 Bertin, E., \& Arnouts, S. 1996, A\&AS, 117, 393

Birkby, J. L., Cappetta, M., Cruz, P., et al. 2014, MNRAS, 440, 1470

Borucki, W. J., Koch, D., Basri, G., et al. 2010, Sci, 327, 977

Bovy, J., Rix, H.-W., Green, G. M., Schlafly, E. F., \& Finkbeiner, D. P. 2016 , ApJ, 818, 130

Brahm, R., Jordán, A., \& Espinoza, N. 2017a, PASP, 129, 034002

Brahm, R., Jordán, A., Hartman, J., \& Bakos, G. 2017b, MNRAS, 467, 971

Brasseur, C. E., Phillip, C., Fleming, S. W., Mullally, S. E., \& White, R. L. 2019, Astrocut: Tools for creating cutouts of TESS images, Astrophysics Source Code Library, ascl:1905.007

Brown, T. M., Baliber, N., Bianco, F. B., et al. 2013, PASP, 125, 1031

Bryan, M. L., Alsubai, K. A., Latham, D. W., et al. 2012, ApJ, 750, 84 Butler, R. P., Marcy, G. W., Williams, E., et al. 1996, PASP, 108, 500 Cardelli, J. A., Clayton, G. C., \& Mathis, J. S. 1989, ApJ, 345, 245

Charbonneau, D., Brown, T. M., Latham, D. W., \& Mayor, M. 2000, ApJL, 529, L45

Claret, A. 2018, A\&A, 618, A20

Claret, A., Hauschildt, P. H., \& Witte, S. 2012, A\&A, 546, A14

Claret, A., Hauschildt, P. H., \& Witte, S. 2013, A\&A, 552, A16

Collier Cameron, A., Wilson, D. M., West, R. G., et al. 2007, MNRAS, 380,1230

Collins, K., Quinn, S. N., Latham, D. W., et al. 2018, AAS Meeting, 231, 439.08

Crane, J. D., Shectman, S. A., \& Butler, R. P. 2006, Proc. SPIE, 6269, 626931 Crane, J. D., Shectman, S. A., Butler, R. P., et al. 2010, Proc. SPIE, 7735, 773553

Crane, J. D., Shectman, S. A., Butler, R. P., Thompson, I. B., \& Burley, G. S. 2008, Proc. SPIE, 7014, 701479

Cupani, G., D'Odorico, V., Cristiani, S., et al. 2018, arXiv:1808.04214

Cushing, M. C., Vacca, W. D., \& Rayner, J. T. 2004, PASP, 116, 362

Dopita, M., Hart, J., McGregor, P., et al. 2007, Ap\&SS, 310, 255

Espinoza, N., Bayliss, D., Hartman, J. D., et al. 2016, AJ, 152, 108

Freudling, W., Romaniello, M., Bramich, D. M., et al. 2013, A\&A, 559, A96 Gaia Collaboration, Brown, A. G. A., Vallenari, A., et al. 2018, A\&A, 616, A1 Gillon, M., Anderson, D. R., Collier-Cameron, A., et al. 2013, A\&A, 552, A82 Guillot, T., Santos, N. C., Pont, F., et al. 2006, A\&A, 453, L21

Hansen, B. M. S., \& Barman, T. 2007, ApJ, 671, 861

Hartman, J. D., \& Bakos, G. Á 2016, A\&C, 17, 1

Hartman, J. D., Bakos, G. Á, Bayliss, D., et al. 2019, AJ, 157, 55

Hartman, J. D., Bakos, G. Á, Torres, G., et al. 2011, ApJ, 742, 59

Hartman, J. D., Bayliss, D., Brahm, R., et al. 2015, AJ, 149, 166

Henry, G. W., Marcy, G. W., Butler, R. P., \& Vogt, S. S. 2000, ApJL, 529, L41 Hippler, S., Bergfors, C., Wolfgang, Brandner., et al. 2009, Msngr, 137, 14

Holman, M. J., Fabrycky, D. C., Ragozzine, D., et al. 2010, Sci, 330, 51

Howell, S. B., Sobeck, C., Haas, M., et al. 2014, PASP, 126, 398

Huang, C. X., Burt, J., Vanderburg, A., et al. 2018, ApJL, 868, L39

Jenkins, J. M., Caldwell, D. A., Chandrasekaran, H., et al. 2010, ApJL, 713, L87

Jenkins, J. M., Twicken, J. D., McCauliff, S., et al. 2016, Proc. SPIE, 9913, 99133E

Johnson, J. A., Gazak, J. Z., Apps, K., et al. 2012, AJ, 143, 111

Jordán, A., Brahm, R., Bakos, G. Á, et al. 2014, AJ, 148, 29

Kaufer, A., \& Pasquini, L. 1998, Proc. SPIE, 3355, 844

Konacki, M., Torres, G., Jha, S., \& Sasselov, D. D. 2003, Natur, 421, 507

Koppenhoefer, J., Saglia, R. P., Fossati, L., et al. 2013, MNRAS, 435, 3133

Kovács, G., Bakos, G., \& Noyes, R. W. 2005, MNRAS, 356, 557

Kovács, G., Bakos, G. Á, Hartman, J. D., et al. 2010, ApJ, 724, 866

Kovács, G., Zucker, S., \& Mazeh, T. 2002, A\&A, 391, 369

Lang, D., Hogg, D. W., Mierle, K., Blanton, M., \& Roweis, S. 2010, AJ, 139,1782

Lightkurve Collaboration, Cardoso, J. V. d. M., Hedges, C., et al. 2018, Lightkurve: Kepler and TESS time series analysis in Python, Version 1.1.1, Astrophysics Source Code Library, ascl:1812.013

Mandel, K., \& Agol, E. 2002, ApJL, 580, L171

Marigo, P., Girardi, L., Bressan, A., et al. 2017, ApJ, 835, 77

Mayor, M., Pepe, F., Queloz, D., et al. 2003, Msngr, 114, 20

Mégevand, D., Zerbi, F. M., Di Marcantonio, P., et al. 2014, Proc. SPIE, 9147, $91471 \mathrm{H}$

Pál, A. 2012, MNRAS, 421, 1825

Penev, K., Bakos, G. Á, Bayliss, D., et al. 2013, AJ, 145, 5

Pepper, J., Kuhn, R. B., Siverd, R., James, D., \& Stassun, K. 2012, PASP, 124, 230

Pepper, J., Pogge, R. W., DePoy, D. L., et al. 2007, PASP, 119, 923 
Pollacco, D. L., Skillen, I., Collier Cameron, A., et al. 2006, PASP, 118, 1407

Price-Whelan, A. M., Sipőcz, B. M., Günther, H. M., et al. 2018, AJ, 156, 123 Queloz, D., Mayor, M., Udry, S., et al. 2001, Msngr, 105, 1

Ricker, G. R., Winn, J. N., Vanderspek, R., et al. 2015, JATIS, 1, 014003

Sestovic, M., Demory, B.-O., \& Queloz, D. 2018, A\&A, 616, A76

Smith, J. C., Stumpe, M. C., Van Cleve, J. E., et al. 2012, PASP, 124, 1000

Stassun, K. G., Collins, K. A., \& Gaudi, B. S. 2017, AJ, 153, 136

Stumpe, M. C., Smith, J. C., Catanzarite, J. H., et al. 2014, PASP, 126, 100

Thorngren, D. P., Fortney, J. J., Murray-Clay, R. A., \& Lopez, E. D. 2016 , ApJ, 831, 64
Torres, G. 2013, AN, 334, 4

Triaud, A. H. M. J., Anderson, D. R., Collier Cameron, A., et al. 2013, A\&A, 551, A80

Tsiaras, A., Waldmann, I. P., Tinetti, G., Tennyson, J., \& Yurchenko, S. N. 2019, NatAs, 3, 1086

Vacca, W. D., Cushing, M. C., \& Rayner, J. T. 2004, PASP, 116, 352

Wakeford, H. R., Sing, D. K., Kataria, T., et al. 2017, Sci, 356, 628

Zacharias, N., Finch, C. T., Girard, T. M., et al. 2013, AJ, 145, 44

Zechmeister, M., \& Kürster, M. 2009, A\&A, 496, 577

Ziegler, C., Law, N. M., Baranec, C., et al. 2018, AJ, 156, 259

Ziegler, C., Tokovinin, A., Briceno, C., et al. 2020, AJ, 159, 19 